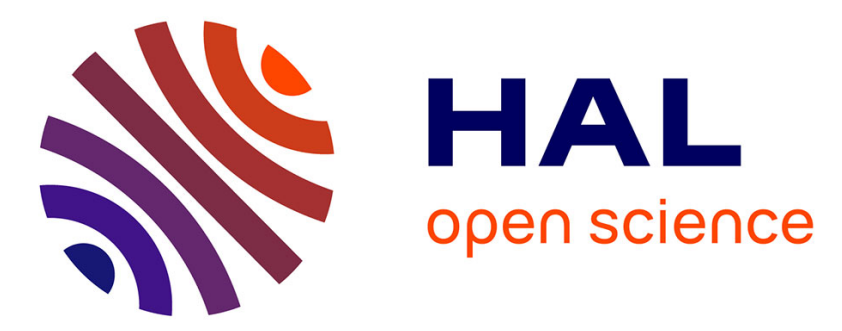

\title{
ID-HALL, a new double stage Hall thruster design. I. Principle and hybrid model of ID-HALL
}

\author{
Loic Dubois, F. Gaboriau, Laurent Liard, Dominique Harribey, Carole \\ Hénaux, Laurent Garrigues, G J M Hagelaar, S. Mazouffre, C. Boniface, \\ Jean-Pierre Boeuf
}

\section{To cite this version:}

Loic Dubois, F. Gaboriau, Laurent Liard, Dominique Harribey, Carole Hénaux, et al.. ID-HALL, a new double stage Hall thruster design. I. Principle and hybrid model of ID-HALL. Physics of Plasmas, 2018, 25 (9), pp.093503. 10.1063/1.5043354 . hal-02326140

\section{HAL Id: hal-02326140 \\ https://hal.science/hal-02326140}

Submitted on 22 Oct 2019

HAL is a multi-disciplinary open access archive for the deposit and dissemination of scientific research documents, whether they are published or not. The documents may come from teaching and research institutions in France or abroad, or from public or private research centers.
L'archive ouverte pluridisciplinaire HAL, est destinée au dépôt et à la diffusion de documents scientifiques de niveau recherche, publiés ou non, émanant des établissements d'enseignement et de recherche français ou étrangers, des laboratoires publics ou privés. 
ID-HALL, a new double stage Hall thruster design. I. Principle and hybrid model of IDHALL

L. Dubois, F. Gaboriau, L. Liard, D. Harribey, C. Henaux, L. Garrigues, G. J. H. Hagelaar, S. Mazouffre, C. Boniface, and J. P. Boeuf

Citation: Physics of Plasmas 25, 093503 (2018); doi: 10.1063/1.5043354

View online: https://doi.org/10.1063/1.5043354

View Table of Contents: http://aip.scitation.org/toc/php/25/9

Published by the American Institute of Physics

\section{PHYSICS TODAY}

MANAGER'S GUIDE

WHITEPAPERS
READ NOW

PRESENTED BY Multiphysics Simulation 


\title{
ID-HALL, a new double stage Hall thruster design. I. Principle and hybrid model of ID-HALL
}

\author{
L. Dubois, ${ }^{1}$ F. Gaboriau, ${ }^{1}$ L. Liard, ${ }^{1}$ D. Harribey, ${ }^{1}$ C. Henaux, ${ }^{1}$ L. Garrigues, ${ }^{1}$ \\ G. J. H. Hagelaar, ${ }^{1}$ S. Mazouffre, ${ }^{2}$ C. Boniface, ${ }^{3}$ and J. P. Boeuf ${ }^{1, a)}$ \\ ${ }^{1}$ LAPLACE, Université de Toulouse, CNRS, INPT, UPS, 118 Route de Narbonne, 31062 Toulouse, France \\ ${ }^{2}$ ICARE, CNRS, Université d' Orléans, $1 C$ avenue de la Recherche Scientifique, 45071 Orléans, France \\ ${ }^{3}$ CNES, 18 Avenue Edouard Belin, 31401 Toulouse, France
}

(Received 8 June 2018; accepted 14 August 2018; published online 6 September 2018)

\begin{abstract}
In Hall thrusters, ions are extracted from a quasineutral plasma by the electric field induced by the local drop of electron conductivity associated with the presence of a magnetic barrier. Since the electric field is used both to extract and accelerate ions and to generate the plasma, thrust and specific impulse are not independent in a Hall thruster. There is a need for versatile thrusters that can be used for a variety of maneuvers, i.e., that can operate either at high thrust or at high specific impulse for a given power. The double stage Hall thruster (DSHT) design could allow a separate control of ionization and acceleration, and hence separate control of thrust and specific impulse. In the DSHT configuration, a supplementary plasma source (ionization stage), independent of the applied voltage, is added and placed upstream of the magnetic barrier (acceleration stage). The DSHT concept is also well adapted to the use of alternative propellants, lighter and with a less efficient ionization than xenon. Several designs of double stage Hall thrusters have been proposed in the past, but these attempts were not really successful. In this paper, we present a brief review of the main DSHT designs described in the literature, we discuss the relevance of the DSHT concept, and, on the basis of simple physics arguments and simulation results, we propose a new design, called ID-HALL (Inductive Double stage HALL thruster). In this design, the ionization stage is a magnetized inductively coupled RF plasma. The inductive coil is inside the central cylinder of the thruster and located nearby the acceleration stage. Preliminary modeling results of this DSHT are described. Published by AIP Publishing. https://doi.org/10.1063/1.5043354
\end{abstract}

\section{INTRODUCTION}

In a Hall thruster [see Fig. 1(a)], the plasma is formed in a channel between two concentric ceramic cylinders. ${ }^{1,2}$ Xenon is injected from the anode side at one end of the channel and is ionized by electrons emitted from an external hot cathode and accelerated by a dc voltage between the cathode and anode. Ions generated in the channel are accelerated through the exhaust plane by this potential drop. In contrast to usual low pressure dc discharges (e.g., magnetron discharge), the potential drop is not localized in an ion sheath next to the cathode but in a well-defined region of the quasineutral plasma. This is possible in a Hall thruster because the cathode is emissive and because of the presence of a radial magnetic field, generated by coils or magnets, which is maximum in the exhaust region. This magnetic field barrier lowers the axial electron conductivity in the exhaust region, leading to an increase in the axial electric field in that region. Electrons emitted by the external cathode are accelerated by this electric field and ionize the gas injected from the anode. The ions, practically insensitive to the magnetic field (Larmor radius larger than the dimensions of the channel) are extracted and accelerated out of the channel by this electric field. The residence time of the electrons in the plasma is considerably increased by the radial magnetic field, allowing

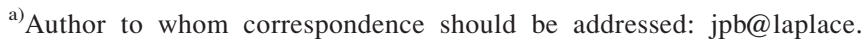
univ-tlse.fr
}

efficient ionization at low gas pressure. The combination of the radial magnetic field and the axial electric field in the channel, near the exhaust plane, leads to a large azimuthal electron current, the Hall current. Electrons are trapped by the magnetic field and only collisions or turbulence can lead to electron transport across the magnetic field barrier.

Figure 1(b) illustrates the physics of a Hall thruster. We see on this figure the axial distributions of the external magnetic field, the axial electric field generated in the quasineutral plasma, and the ionization rate in the region upstream of the large electric field region. The relative positions of the ionization region and acceleration region (large electric field region) certainly play an important role in the effective extraction of the ions from the plasma and depend on the magnetic field distribution.

Cross-field electron transport is controlled by collisions with neutral atoms and with the channel walls as well as by instabilities and turbulence associated with the large Hall electron current (see Refs. 1 and 2 and references therein). It therefore strongly depends on the magnetic field profile. The role of instabilities or electron-wall collisions on cross-field electron transport is not completely understood and the optimization of the magnetic field profile is still rather empirical.

In the single stage Hall thruster of Fig. 1, the electric field is responsible for ion extraction and acceleration as well as for ionization (electrons entering the channel from the cathode side gain energy from this field and ionize the 


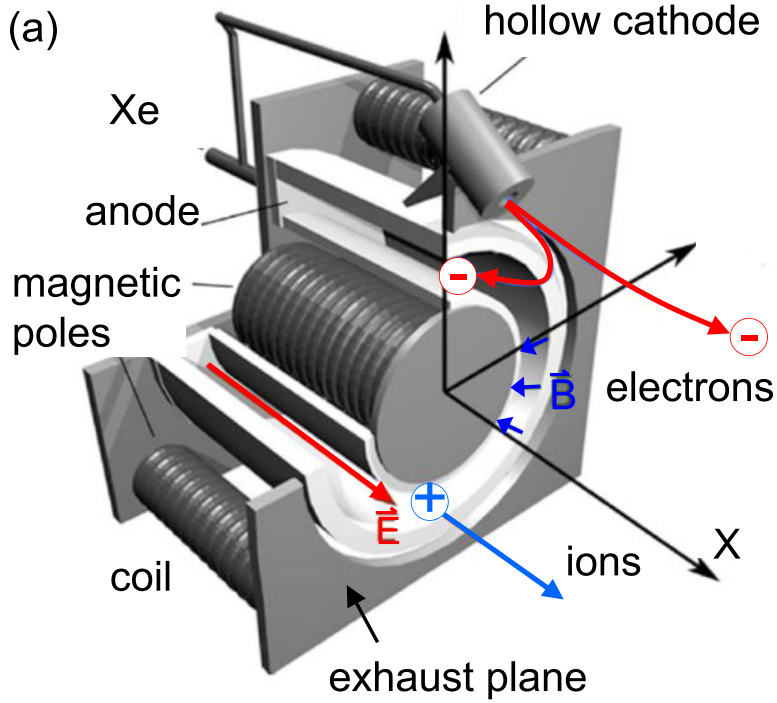

(b)

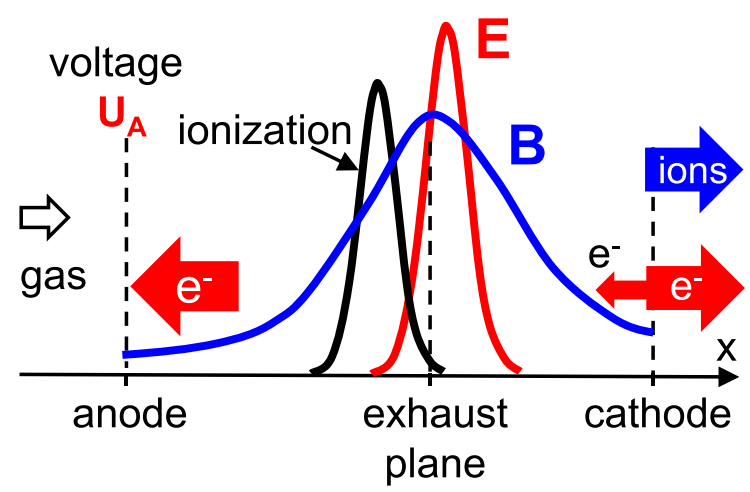

FIG. 1. (a) Schematic of a Hall thruster; (b) simplified representation of the (time averaged) axial distributions (along a mid-channel axis) of the radial magnetic field $\mathrm{B}$, axial electric field $\mathrm{E}$, and ionization rate in a Hall thruster. A large part of the electron current emitted by the cathode is used to neutralize the extracted ion beam.

flow of neutral atoms emitted on the anode side). This implies that specific impulse (i.e., ion velocity) and thrust are not independent.

One way to allow separate control of ionization and acceleration is to add a plasma source upstream of the magnetic barrier, whose power can be adjusted independently of the discharge voltage. Such a design is called a double stage Hall thruster (DSHT) where the plasma source forms the ionization stage and the magnetic barrier in the thruster channel is the acceleration stage. In an ideal double stage Hall thruster (DSHT), ionization would be completely controlled by adjusting the mass flow rate and power deposition in the first stage (ionization stage), while acceleration would be controlled by the voltage applied across the magnetic barrier. However, in a DSHT, electrons emitted by the external cathode are still required to neutralize the extracted ion beam in the channel. These electrons gain energy in the large electric field of the acceleration stage and can therefore also contribute to ionization of the gas flow. Therefore, ionization is not entirely controlled by the plasma source of the ionization stage as would be the case in an ideal DSHT concept. To minimize the contribution of these electrons to ionization and to limit the total power they absorb, the magnetic field must be adjusted to minimize the current of electrons entering the channel. This is not a simple task since cross-field electron transport in the magnetic barrier is "anomalous" and cannot be easily controlled.

Operation of a Double-stage Hall thruster seems especially relevant in three cases, namely: (i) high propellant mass flow rate and low acceleration voltage, (ii) low mass flow rate and high voltage, and (iii) operation with light gases (mass below the xenon mass) at moderate specific impulse. In all cases, full ionization of the injected gas flow cannot be achieved. Notice cases (i) and (ii) correspond to high thrust and high specific impulse operation, respectively.

Whether or not the ideal way of operation of a DSHT can be achieved or can lead to good performance is one question that must be addressed when designing DSHTs. Another important question is how to optimize the extraction through the magnetic barrier of all ions generated in the first stage. The extraction area, $\pi\left(R_{2}^{2}-R_{1}^{2}\right)$, where $R_{1}$ and $R_{2}$ are the inner and outer radii of the channel, can be small with respect to the total area of the first stage so that it is essential to efficiently limit wall losses in the ionization chamber, e.g., by using magnetic cusps. It is also intuitive that ionization in the first stage should be such that the maximum plasma density is as close as possible to the magnetic barrier so that a significant fraction of positive ions can be extracted.

A number of DSHT designs have been proposed and tested along the years. However, the performance of these thrusters has not really reached expectations. In particular, it is not clear, reading the literature on DSHTs, if they really operated efficiently in regimes where most of the extracted ions were actually generated in the ionization stage (and not in the acceleration stage).

In Sec. II, we present a non-exhaustive review of previous DSHT concepts. The requirements for an efficient DSHT concept are discussed in Sec. III, and a new design based on these requirements and called ID-HALL (Inductively coupled Double stage HALL thruster) is described in Sec. IV. The hybrid model used to describe the properties of IDHALL is summarized in the first part of Sec. V and the results showing single stage and double stage operation, as well as a parametric study of the performance are discussed in the other parts of Sec. V. Finally, Sec. VI presents a sensitivity analysis of the results on the anomalous transport coefficients describing cross-field electron transport.

Paper $\mathrm{II}^{3}$ presents some experimental characterization of the first stage of ID-HALL.

\section{NON-EXHAUSTIVE REVIEW OF PREVIOUS DSHT DESIGNS}

In some DSHT concepts, the ionization stage is outside and upstream of the thruster channel) while in other concepts. the ionization and acceleration stage are both in the thruster channel. Examples of plasma with the ionization stage outside of the channel are shown and briefly described below: "electron bombardment" sources, i.e., using energetic electrons from a hot cathode in a multicusp chamber (Fig. 2) 


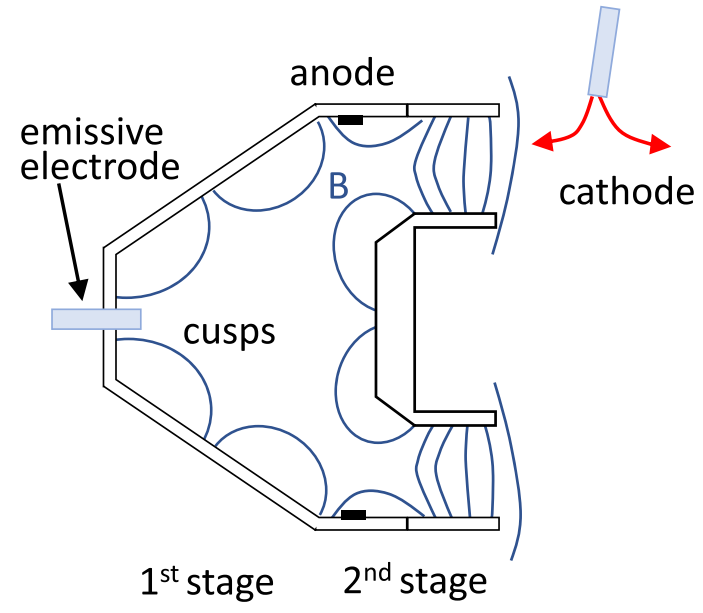

FIG. 2. DSHT with an electron bombardment cusped ionization stage (similar to the NASA-173GT developed by Peterson ${ }^{4}$ ).

or in a more complex magnetic configuration (Galathea design, Fig. 3), ECR (Electron Cyclotron Resonance, Fig. 4), helicon (Fig. 5), and microwave source (Fig. 6).

In the DSHT with multicusp electron bombardment ionization source (see, e.g., the NASA 173-GT, studied by Peterson ${ }^{4}$ ) and shown in Fig. 2, the plasma is generated by electrons of a few tens of $\mathrm{eV}$ emitted by an emissive cathode in the first stage. This source is very similar to an electron bombardment gridded ion source ${ }^{1}$ where the extracting grids are replaced by a magnetic barrier.

The experimental results on the NASA-173GT described by Peterson ${ }^{4}$ were disappointing since operations in a single or double-stage mode did not appear to be very different. One could expect that the relative contributions to the overall ionization of the electron source inside the chamber or of electrons coming from the external cathode would be affected by the mass flow rate, applied voltage, or injected electron currents, leading to different properties of the thruster in the double and single stage modes. However, there was no clear conclusion in Ref. 4 on the different (a)

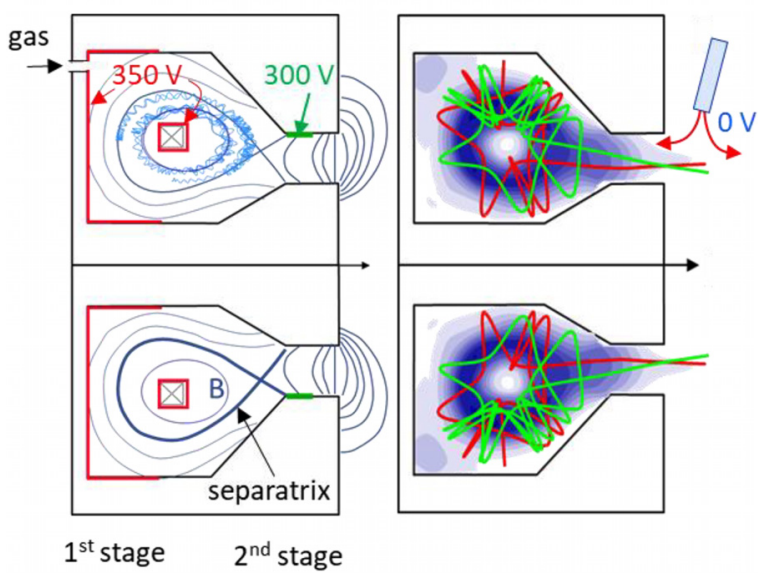

FIG. 3. Galathea concept of Morozov et al. Typical electron, (a), and ion, (b) trajectories from simulations ${ }^{8,10}$ are displayed. The grey levels in (b) correspond to calculated equipotential contours.

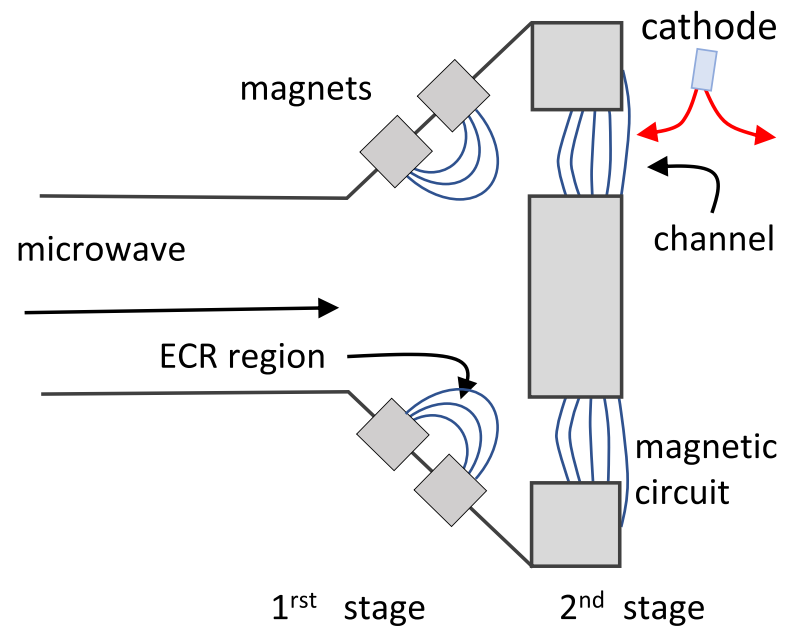

FIG. 4. DSHT with an Electron Cyclotron Resonance (ECR) ionization stage. $^{14}$

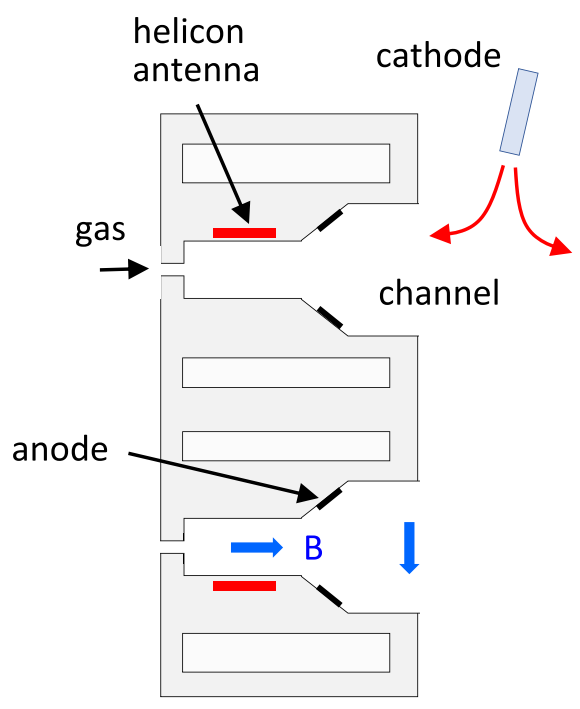

$1^{\text {st }}$ stage $2^{\text {nd }}$ stage

FIG. 5. DSHT with an helicon ionization stage (see, e.g., Martinez et al. ${ }^{15}$ ).

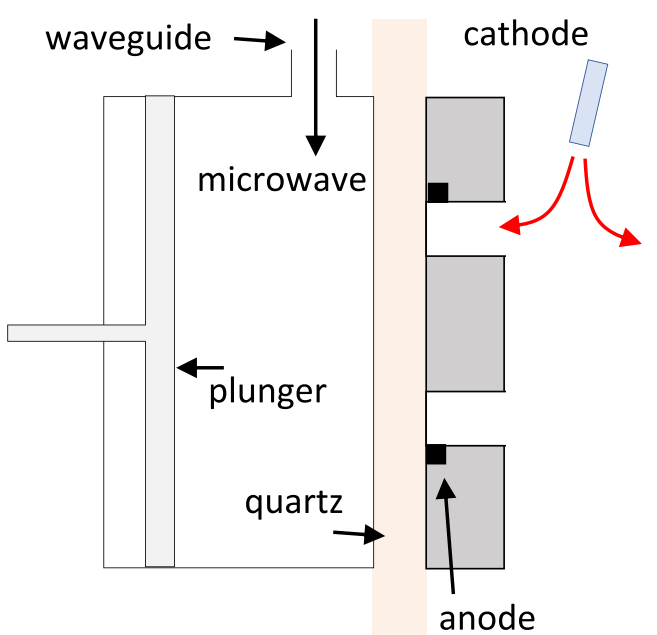

FIG. 6. DSHT with a microwave ionization stage. ${ }^{19,23}$ 
physics involved in the single stage and double-stage modes or on the advantage of operating in a double-stage mode.

The SPT-MAG is a very interesting and appealing DSHT concept proposed by Morozov and Bugrova, ${ }^{5,6}$ and based on a semi-galathea magnetic field configuration. The galathea design is an efficient magnetic confinement configuration proposed by Morozov and Savelyev ${ }^{7}$ in the 1990s. Figure 3 displays the SPT-MAG DSHT design and illustrates the magnetic electron confinement and electrostatic ion confinement obtained in this configuration.

A voltage drop of several tens of volts is applied between an intermediate anode placed in the first stage at the entrance of the channel, and the anode around the myxina and on the chamber wall. The intermediate anode plays the role of an anode for the acceleration stage, and of a cathode for the ionization stage. Electrons are trapped around the magnetic field lines [see some electron trajectories in Fig. 3(a)]. Because the magnetic field lines are quasi-equipotential while the electron conductivity perpendicular to the magnetic field is low, the applied voltage in the source is distributed between the separatrix line (see Fig. 3) at a potential close to that of the intermediate anode, and the anode. This leads to the formation of a potential well that traps the ions electrostatically and guides them to the channel entrance. Typical ion trajectories are shown in Fig. 3(b).

The voltage drop in the potential well also provides energy to the electrons in their cross-field motion from the separatrix line to the anode, leading to ionization and plasma generation in the source. The electron and ion trajectories in Fig. 3 are deduced from a hybrid simulation. ${ }^{8-10}$ In this model, the description of ion transport is kinetic while the description of electron is fluid. Ionization is however deduced from a Monte Carlo simulation of electron transport.

Experimental results on the SPT-MAG ${ }^{11}$ showed that this DSHT could operate in a relatively large range of conditions, at high thrust (e.g., $190 \mathrm{mN}$ at $300 \mathrm{~V}$ and $9 \mathrm{mg} / \mathrm{s}$ xenon mas flow rate) or high specific impulse (e.g., $3650 \mathrm{~s}$ at $900 \mathrm{~V}$ and $3 \mathrm{mg} / \mathrm{s}$ xenon mass flow rate). Although this is an interesting result, it is not clear whether this thruster really operated as a DSHT, i.e., if the plasma in the chamber was generated by electrons heated by the applied electric field in the ionization stage or by energetic electrons coming from the external cathode (which corresponds to single stage operation). This is related to another important question concerning the role of the intermediate electrode, i.e., the cathode of the ionization chamber. The simulations ${ }^{10}$ showed that this electrode must be emissive to create the potential well in the ionization chamber (otherwise the potential drop takes place in the sheath of the intermediate electrode). Without electron emission and potential well, the first stage does not operate as a separate source of ionization and ionization in the chamber, if present, is only due to electrons coming from the external cathode. In the experiments reported in the literature, the intermediate electrode was not emissive. The paper by Chesta et $a l .{ }^{9}$ indicates that "the possibility to include an emitting internal electrode (hollow cathode) in the ionization stage has been carefully assessed and finally rejected". The authors did not explain this choice which is not consistent with ideal DSHT operation and model predictions. The question of ion extraction from the ionization stage in a DSHT similar to that of Fig. 3 (with a non-emissive intermediate electrode) is discussed in the experimental and numerical work of $\mathrm{Yu}$ et $a .^{12,13}$ Their results show that the magnetic field intensity in the ionization stage must be carefully optimized to generate the potential well and efficiently extract the ions. We can conclude that although the SPTMAG is a smart concept, its magnetic field design is rather complex and its efficient operation as a DSHT is still to be demonstrated. Moreover, we did not mention the possibility of turbulence and anomalous transport in the ionization stage that could prevent the formation of potential well.

Microwave plasma sources operating at Electron Cyclotron Resonance (ECR) are also possible candidates for the ionization stage. A DSHT using an ECR ionization stage has been proposed by Molina-Morales et al. ${ }^{14}$ and is shown in Fig. 4. The microwave field is injected axially from the back of the ionization stage and the resonance region is circular, between two ring magnets located a few $\mathrm{cm}$ behind the entrance of the Hall acceleration channel. In the experiments of Molina-Morales, the microwave field frequency was $4.25 \mathrm{GHz}$. For this frequency, the resonance magnetic field $B_{R}$, defined by $\Omega_{c e, R}=\omega\left(\Omega_{c e, R}=e B_{R} / m\right.$ is the electron cyclotron angular frequency and $\omega$ the wave angular frequency) is on the order of $150 \mathrm{mT}$. This magnetic field is much larger than the magnetic field of the magnetic barrier of a Hall thruster (typically $15 \mathrm{mT}$ ). Since the resonance region and the acceleration region are relatively close together $(\mathrm{a}$ few $\mathrm{cm})$ in the design of Fig. 4, the magnetic field of the acceleration stage was strongly perturbed by the magnetic field of the resonance region, which was detrimental to a correct operating of the magnetic barrier. The authors of Ref. 14 also mentioned that in this ECR design, the microwave propagation is cut-off at the critical density defined by $\omega_{p e}=\omega\left(\omega_{p e}\right.$ is the electron plasma angular frequency) which corresponds to $2.2 \times 10^{11} \mathrm{~cm}^{-3}$ for a frequency of $4.25 \mathrm{GHz}$. The plasma density under typical Hall thrusters conditions ${ }^{2}$ is on the order $10^{12} \mathrm{~cm}^{-3}$ or more so the plasma density in this ECR ionization source was not large enough for satisfactory operation. The ECR DSHT concept was not further explored due to the limitations discussed above.

Attempts at developing helicon DSHTs have been reported in Refs. 15-17, with a design schematically represented on Fig. 5. Here again, the results did not show an efficient operation in a DSHT regime.

For example, in most results of Ref. 17, the increase in the measured thrust with helicon power was very small, typically less than $10 \mathrm{mN}$ per $\mathrm{kW}$ of the helicon source, for a DSHT operating with a thrust around $200 \mathrm{mN}$ with the helicon source turned OFF.

An important issue in the helicon DSHT design is the question of the connection between the axial magnetic field of the helicon and the radial magnetic field of the acceleration stage. No details on the magnetic field configuration were given in the references above.

Note also that Harada et al. ${ }^{18}$ demonstrated "electrostatic acceleration from a helicon plasma using a cusped magnetic field," with an interesting design that could be called the "helicon double-stage cusped-field thruster" or "helicon 
double-stage cylindrical Hall thruster." They were able to extract ions from the helicon plasma under conditions where the cathode-anode voltage was not sufficient to sustain the plasma (proving that the thruster was really operating in a double-stage mode). The performances of this thruster were however not measured.

In the DSHT designs above, the ionization stage is clearly separated from the acceleration stage in the thruster channel. In some DSHT designs, the ionization stage can be inside the channel as shown in the examples below.

In the DSHT of Fig. 6, a microwave field is injected in a cylindrical cavity-resonator behind the channel. The $5.8 \mathrm{GHz}$ microwave field enters the channel through a quartz window and propagates in the acceleration channel. The length of the cylindrical cavity-resonator can be optimized with a plunger. According to the authors of Ref. 19, the microwave propagates along the dielectric-plasma interfaces, i.e., the plasma is excited in a surface-wave (SW) mode, allowing plasma densities above the critical density.

Measurements on this surface-wave double-stage Hall thruster (SW DSHT) showed that this thruster could operate in a double-stage regime. For example it was possible to extract ions through the magnetic barrier even for very low voltage between cathode and anode, i.e., without significant ionization due to electrons emitted by the external cathode and entering the channel. The ion current at these low voltages was however small, about ten times lower than the current corresponding to full ionization of the injected gas flow. An interesting feature of this SW DSHT is that ionization due to the surface wave electron heating takes place very close to the magnetic field barrier. Ions generated in this region can therefore be efficiently extracted and accelerated by the axial electric field before they are lost to a wall. The study of a DSHT with RF electron heating at $13.56 \mathrm{MHz}$ in an ATON type plasma source was also reported in Ref. 20. The results showed that an additional RF power of $100 \mathrm{~W}$ for a $600-900 \mathrm{~W}$ thruster could increase the propellant ionization and thrust by about $5 \%$.

Another example of DSHT with the ionization stage inside the channel is the double peaked magnetic field design of Fig. 7..$^{21,22}$ In this concept, the ionization stage consists of

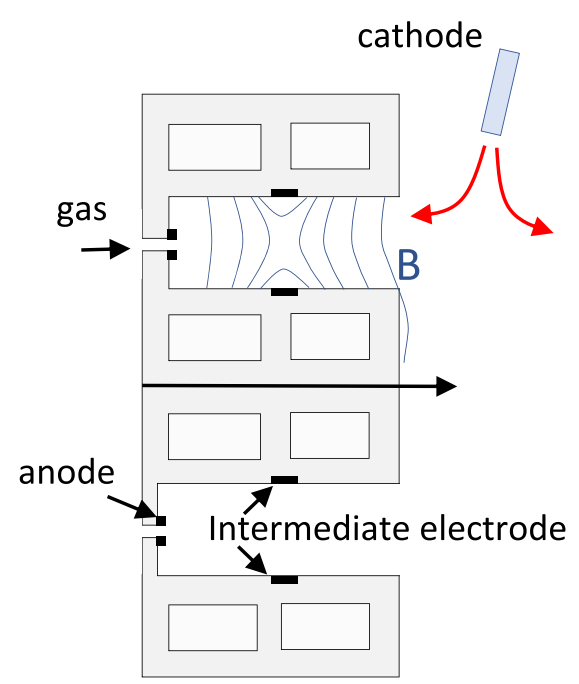

FIG. 7. DSHT with a double peaked magnetic field in the channel. ${ }^{21,22}$ another magnetic barrier placed between an intermediate electrode and the anode, upstream of the magnetic barrier of the acceleration stage. A voltage is applied between the intermediate electrode and the anode and this voltage can be controlled independently from the accelerating voltage. This concept implies a relatively long channel $(5 \mathrm{~cm})$. Simulations $^{22}$ showed that electron-ion recombination at the channel walls was enhanced and that a non-negligible part of the total ionization was actually taking place in the acceleration stage.

\section{RELEVANCE OF THE DSHT CONCEPT}

The different attempts at designing DSHTs have not been very convincing so far. In this section, we try to analyze the reasons for this limited success.

As mentioned above, a first issue is that electrons emitted by the external cathode and gaining energy in the acceleration region are still present in a DSHT and can contribute to ionization of the gas flow. Operation in a double-stage mode at high voltage is therefore questionable since most of the ionization can be performed by these electrons unless there is a way to limit or control the current of electrons entering the channel from the cathode side. This would need a complete reconsideration of the magnetic field design in the acceleration stage. If the magnetic field in the acceleration stage is kept the same as in a single stage thruster, operation in a double-stage mode would be more useful at low voltages, i.e., under conditions where ionization provided by the electrons emitted by the external cathode would not be sufficient to sustain the plasma. In that case, the ionization stage would fully play its role and the thrust of the DSHT could be adjusted by changing the power deposited in the ionization stage and the mass flow rate of the injected gas, independent of the accelerating voltage. This type of operation is well-suited to the use of lighter gases such as argon where the same ion velocity or specific impulse as in xenon can be achieved at lower voltages.

A second issue is that efficient operation of a DSHT is possible only if most of the ions generated in the ionization stage can be extracted through the magnetic barrier (and not lost at the walls). In a gridded ion thruster, a large part of the ions generated in the plasma source can be efficiently extracted and accelerated through the grids. The physics of ion extraction through a magnetic barrier is much more complicated since extraction is possible only if electrons from the external cathode can themselves cross the magnetic barrier to neutralize the ion beam. The different attempts at designing DSHTs have been very empirical and consisted in connecting a plasma source to the channel of a single stage Hall thruster. It seems, however, that the position of the ionization region with respect to the acceleration region is an important aspect of Hall thrusters. In single stage Hall thrusters, these two regions overlap, as shown in Fig. 1(b), minimizing the wall losses and allowing efficient ion extraction by the axial field.

Another important point is that the extraction area of a DSHT, defined by the annular section of the channel may be small with respect to the total wall area of the ionization 
stage. Charged particle losses at the wall are therefore important and must be limited by magnetic confinement. The SPTMAG of Fig. 3 shows a nice example of plasma confinement by the magnetic field but the ionization region is far from the acceleration region and ions can be lost in the region of the intermediate electrode and at the channel walls.

We conclude from this discussion that an efficient DSHT design requires that the ionization region be very close to (and, if possible, overlapping with) the acceleration region. The ID-HALL DSHT described in Sec. IV has been designed to move closer to this goal.

\section{A NEW DSHT DESIGN: ID-HALL}

ID-HALL (patent pending) is an Inductively coupled Double-Stage HALL thruster schematically represented in Fig. 8.

The ID-HALL design combines the concentric cylinder configuration of a single stage Hall thruster with an efficient

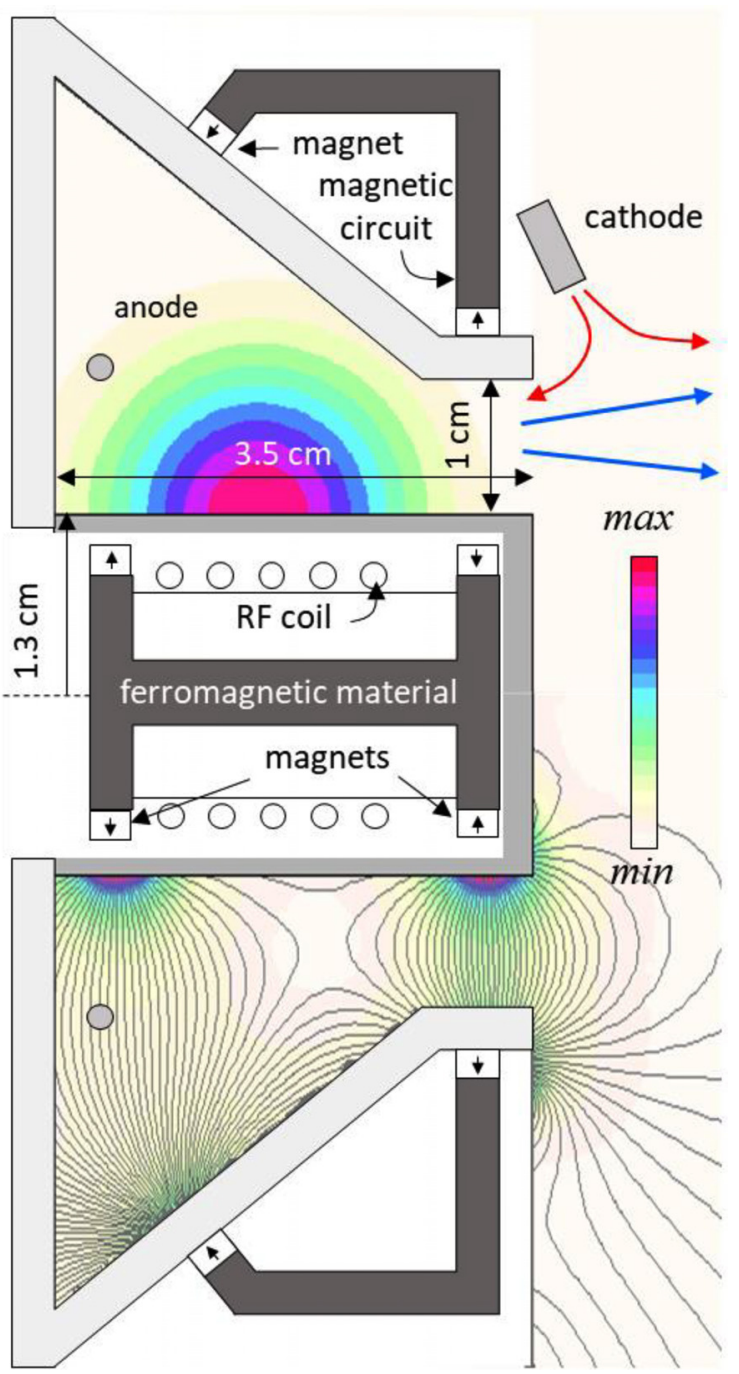

FIG. 8. The ID-Hall Double-Stage Hall Thruster. The inner cylinder is made of quartz and the other walls in the ionization chamber and in the channel are made of ceramic material. The $2 \mathrm{D}$ plots represent the magnetic field distribution (bottom; color plot: field intensity, lines: magnetic field lines) and the assumed spatial distribution of the power absorbed per electron, $\theta_{R F}(x, r)$ (top). magnetized inductively coupled RF plasma source whose coil is placed inside the inner cylinder.

Figure 8 shows a schematic representation of the IDHALL thruster together with plots of the magnetic field distribution and of the assumed distribution of the RF power absorbed per electron (see Sec. V).

It has been shown ${ }^{24,25}$ that a plasma generated by an internal coil with a central cylindrical magnet can reach high densities and is very well localized in the form of a torus around the cylindrical coil. This is because electrons are moving back and forth along the axial magnetic field, while being heated by the azimuthal electric field induced by the inductive coil. Figure 9 shows the light emitted by the inductively coupled magnetized plasma generated in argon around the inner cylinder of ID-HALL (the magnetic field here is only generated by a cylindrical magnet placed inside the coil, i.e., the RF ionization source is separated from the rest of the thruster).

The toroidal plasma density profile obtained with this configuration of inductively coupled magnetized plasma source is very well adapted to the geometry of a Hall thruster since the plasma can be localized close to the entrance of the thruster channel.

The axial component of the magnetic field around the inner cylinder plays an important role in trapping the electrons in the region where they can be heated by the azimuthal RF field induced by the coil.

In the more complex magnetic configuration of IDHALL, the axial magnetic field generated by the internal magnets is connected to the radial magnetic field of the magnetic barrier in the acceleration channel. In order to confine the plasma as much as possible and to limit the charged particle losses to the walls, the magnetic circuit is designed so

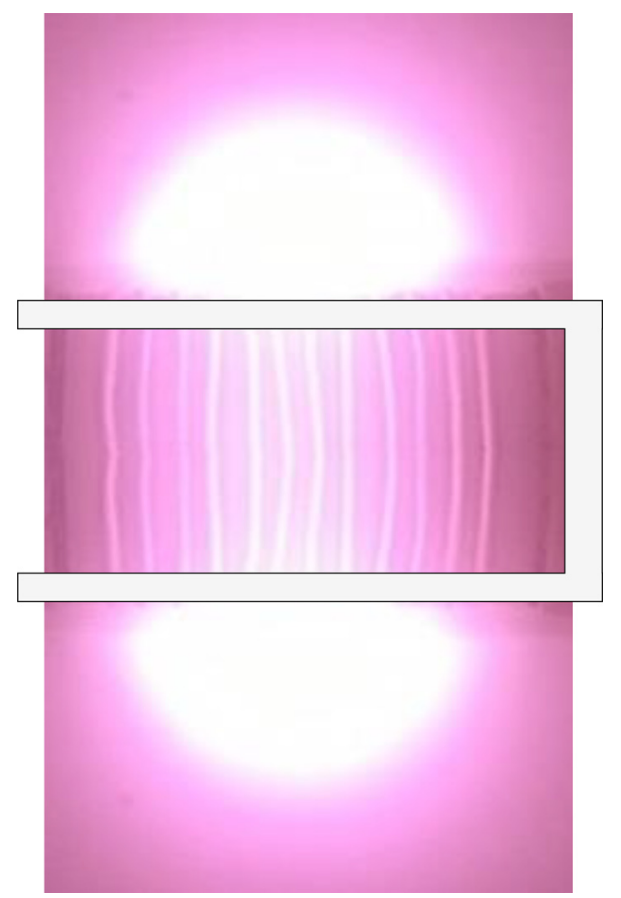

FIG. 9. Photo of the toroidal plasma formed in argon around the inner cylinder of ID-HALL. The outer part of the magnetic circuit is not present (the magnetic field is generated by a cylindrical magnet placed inside the coil). 
that the magnetic field has components parallel to the walls. This implies the presence of magnetic cusps in the ionization chamber (Fig. 8). The magnetic field is generated by magnets indicated by the short arrows in white rectangles in Fig. 8. The magnetic circuit is represented by the darker grey region. The part of the magnetic circuit inside the coil in the inner cylinder is made of ferrite material to allow a good coupling of the RF inductive power. The conical profile of the outer-wall of the ionization stage allows to have a larger plasma volume around the zero-magnetic field region. The anode position must be chosen to avoid a large potential drop between the anode and plasma. The plasma potential may be below the anode potential because of the second magnetic field barrier in the back of the ionization chamber. This question is briefly discussed in the modeling results below. Another issue is to minimize the development of eddy currents in the anode from the RF coupling. The optimum position and size of the anode will be determined experimentally and discussed in a forthcoming paper.

A magnetic configuration of the magnetic barrier similar to that of a magnetically shielded thruster ${ }^{26,27}$ is also possible and will be tested in the near future.

\section{PRINCIPLES OF THE MODEL AND SIMULATION RESULTS}

A first prototype of the ID-HALL DSHT has been built and is currently being tested. A Paper $\mathrm{II}^{3}$ presents some experimental characterization of the inductive plasma source of the ionization stage.

We present here some preliminary simulation results which have been obtained with a hybrid fluid-particle simulation model (the HALLIS software ${ }^{28}$ ) whose principles are identical to the model described in Refs. 10, 29, and 30. In a first part, we briefly recall the principles of the model. We then present simulations of ID-HALL operation in single stage and double stage regimes.

\section{A. Summary of the model}

The model is two-dimensional (2D) in the radial-axial, $(\mathrm{r}, \mathrm{x})$ plane. Electrons are treated as a fluid while ions and neutral atoms are described as particles (kinetic description). The model is quasi-neutral.

Knowing the electric field $E$ and ionization source term $n_{a} k_{i}\left(T_{e}\right)$ at the beginning of a time step $\left(n_{a}\right.$ is the atom density and $k_{i}\left(T_{e}\right)$ the ionization rate deduced from the ionization cross section for a Maxwellian electron distribution of temperature $T_{e}$ ), the ion super-particles are moved for one time step according to the electric field force and taking into account particle generation by ionization and losses to the walls.

The plasma density $n$ and ion flux $\Gamma_{i}=n v_{i}$ are known at the end of the time step ( $v_{i}$ is the ion mean velocity). Atoms are moved for one time step taking into account wall collisions (with semi-isotropic scattering by the walls), ionization, and (semi-isotropic) atom generation at the walls due to ion recombination with electrons.

Knowing the plasma density and electric field, and assuming a drift-diffusion electron flux, the new electron temperature is then obtained by integrating the electron energy equation over one time step assuming a Maxwellian electron distribution $\left(\varepsilon_{e}=\frac{3}{2} k T_{e}\right.$ is the electron mean energy) and neglecting the directed energy with respect to the thermal energy

$$
\begin{gathered}
\frac{\partial n \varepsilon_{e}}{\partial t}+\frac{5}{3} \nabla \cdot\left(\Gamma_{e} \varepsilon_{e}\right)-\frac{10}{9} \nabla \cdot\left(\mu_{e} n \varepsilon_{e} \nabla \varepsilon_{\mathrm{ee}}\right) \\
=-E \cdot \Gamma_{e}+n \theta_{R F}-n_{a} n \kappa-n W .
\end{gathered}
$$

$\theta_{R F}(x, r)$ is the power absorbed per electron due to the RF coupling in the ionization chamber (see below), $\kappa$ is the energy loss rate due to collisions with atoms (deduced from the electron-atom cross-sections and electron temperature), and $W$ corresponds to the energy losses to the channel walls. The electron mobility $\mu_{e}$ is a tensor.

The new electric field at the end of the time step is obtained from the combination of the current continuity equation $\nabla \cdot\left(\Gamma_{i}-\Gamma_{e}\right)=0$ and the electron momentum equation in the drift-diffusion form (inertia is neglected) $\Gamma_{e}=-n \mu_{e} E-\mu_{e} \nabla\left(n T_{e}\right)$. This gives for the electric potential $\Phi: \nabla \cdot\left(n \mu_{e} \nabla \Phi\right)=-\nabla \cdot\left[\Gamma_{i}-\mu_{e} \nabla\left(n T_{e}\right)\right]$ which can be solved for $\mathrm{E}$ knowing the plasma density, ion flux, electron temperature, and electron mobility (including the contribution of anomalous transport). The electron mobility is not isotropic (mobility is much smaller in the direction perpendicular to the magnetic field than in the direction parallel to B), so that the temperature and electric field equations are actually tensor equations.

The equation for the electric potential above is a strongly anisotropic elliptic equation. The anisotropy of the equation is related to the Hall parameter (ratio of electron cyclotron angular frequency to the effective electron collision frequency) which can be as large as $10^{2}-10^{3}$ in a Hall thruster. To simplify the numerical solution of this equation, the direction perpendicular and parallel to the magnetic field lines are treated separately. In the direction parallel to the magnetic field lines, we use the so-called "Morozov approximation," i.e., the electric force in the electron momentum equation is supposed to balance the pressure gradient (the mobility perpendicular and parallel to $\mathrm{B}$ are noted $\mu_{\perp}$ and $\mu_{/ /}$, respectively):

$\Gamma_{e, / /}=-n \mu_{e, / /} E_{/ /}-\mu_{e, / /} \nabla_{/ /}\left(n T_{e}\right) \approx 0$, which gives, for the potential: $\Phi(x, r)=\Phi^{*}(\lambda)+T_{e}(\lambda) \ln \left(n / n_{0}\right)$, where $\lambda$ is constant along a magnetic field line, i.e., is a stream function deduced from the magnetic field distribution

$$
\partial_{x} \lambda=r B_{r} ; \quad \partial_{r} \lambda=-r B_{x} .
$$

The problem then becomes one-dimensional in the direction perpendicular to the magnetic field where the perpendicular electron flux can be written as

$$
\Gamma_{e, \perp}=r B \mu_{\perp} n \partial_{\lambda} \Phi-r B \mu_{\perp} \partial_{\lambda}\left(n T_{e}\right) .
$$

Integrating this equation along the magnetic field lines and using current continuity (with the known ion flux), one gets a first order differential equation for the potential $\Phi$.

It is well known that the collisional electron mobility perpendicular to the magnetic field is not sufficient to explain 
the experimental results, and, as in most hybrid models of Hall thrusters, we use effective electron collision frequencies to describe the role of turbulence or electron-wall interaction on electron transport across the magnetic field. These effective collision frequencies are defined by adjustable coefficients as described in previous studies ${ }^{29-32}$ and summarized below. The effective momentum transfer collision frequency used in the electron mobility perpendicular to the magnetic field is taken as

$$
\nu=n_{a} k_{\mathrm{m}}+\nu_{\mathrm{C}}+\nu_{\mathrm{w}}+\nu_{B}
$$

where $k_{m}$ is the momentum collision rate take constant and equal to $2.5 \times 10^{-13} \mathrm{~m}^{3} \mathrm{~s}^{-1}$ for xenon, $\nu_{\mathrm{C}}$ the electron-ion Coulomb collision frequency, $\nu_{\mathrm{w}}$ a collision frequency describing the effect of electron-wall interaction, and $\nu_{B}$ a collision frequency representing the effect of turbulence.

$\nu_{\mathrm{w}}$ and $\nu_{B}$ are written, respectively, as

$$
\nu_{\mathrm{w}}=\alpha \times 10^{7} \mathrm{~s}^{-1} \quad \text { and } \quad \nu_{\mathrm{B}}=\frac{\beta}{16} \frac{e B}{m_{e}} .
$$

In most of the simulations below the adjustable $\alpha$ and $\beta$ coefficients are taken as: $\alpha=1$ and $\beta=0$ inside the channel and in the ionization chamber, $\alpha=0$ and $\beta=1$ outside the channel. With these assumptions, the effective collision frequency and electron mobility outside the channel are much larger than inside the channel (see, e.g., the discussion in Ref. 33) with a discontinuity at the exhaust plane. The sensitivity of the results to the assumptions on anomalous transport is discussed in Sec. VI where the different electron collision frequencies ("real" and "anomalous") are plotted for two typical cases.

In the electron energy equation above, the collisional energy loss coefficient (units $\mathrm{eV} \mathrm{m}^{-3} \mathrm{~s}^{-1}$ ), $\kappa$, is obtained from look-up tables as a function of $\varepsilon_{e}=\frac{3}{2} k T_{e}$. The energy loss term due to electron wall scattering in the electron energy equation above is of the form $n W$, where $W=\varepsilon_{e} \nu_{\varepsilon}$ and $\nu_{\varepsilon}$ is an energy loss frequency. This energy loss frequency can be deduced from sheath models taking into account secondary electron emission by the walls (see Ref. 2 and references therein). There are still questions about the accuracy of these models ${ }^{2}$ but the most standard models give energy loss frequencies on the order of $10^{7} \mathrm{~s}^{-1}$ in the secondary emission saturation regime. In this section, we use an empirical energy loss frequency due to electron-wall interaction of the form $\nu_{\varepsilon}=\delta \times 10^{7} \exp \left(-U / \varepsilon_{e}\right) \mathrm{s}^{-1}$ with $\delta=0.5$ inside the channel and ionization chamber and $\delta=0$ outside the thruster, and $U=20 \mathrm{eV}$. The sensitivity to this assumption is also discussed in Sec. VI.

The values of the anomalous transport parameters above are consistent with the values used in the simulations of other thrusters but they have not yet been adjusted to fit experimental measurements on the ID-HALL thruster.

The sheath potential at the walls is not calculated in the model (this is not necessary since an empirical expression of the electron energy losses to the walls is used) and the voltage applied between the cathode and anode does not take into account the possible potential drop in the anode sheath.
We also neglect multiply charged ions and ion-neutral collisions.

The RF coupling in the ionization chamber is described in a simple way in the model by imposing a given total RF power, $P_{R F}$, absorbed by the electrons, with a given spatial distribution of the power density absorbed per electron, $\theta_{R F}(x, r)$ (appearing in the heating term of the electron energy equation above). The spatial profile of $\theta_{R F}$ used in the simulations below is a Gaussian function of $x$ and $r$, and is shown in Fig. 8.

This distribution is consistent with a skin depth of about $5 \mathrm{~mm}$, corresponding to a plasma density of $10^{18} \mathrm{~m}^{-3}$. A more detailed study of the consequence of this spatial profile on the results will be presented in a future paper.

$\theta_{R F}$ is normalized at each time step of the simulation to maintain a constant absorbed power, by writing

$$
P_{R F}=\iiint_{V} \theta_{R F}(x, r) n_{e}(x, r) 2 \pi r d r d x .
$$

\section{B. Simulations results in a single stage regime}

In this section, we study the operation of ID-HALL in a single stage regime, i.e., when the RF source is $\mathrm{OFF}$ $\left(\mathrm{P}_{\mathrm{RF}}=0\right)$, for an applied $\mathrm{DC}$ voltage $\mathrm{V}_{\mathrm{DC}}=250 \mathrm{~V}$ and $\mathrm{a}$ xenon mass flow rate of $2 \mathrm{mg} / \mathrm{s}$. Xenon is injected uniformly (semi Maxwellian flux at a temperature of $500 \mathrm{~K}$ ) along the back plate of the ionization chamber.

In these conditions, the calculated current does not present low frequency oscillations. The total current $I_{T}$ is $1.98 \mathrm{~A}$, to be compared with an ion current of $1.47 \mathrm{~A}$ corresponding to the full ionization of $2 \mathrm{mg} / \mathrm{s}$ of xenon.

The current in the exhaust plane is composed of the ion beam current plus the current of electrons entering the channel. The calculated ion beam current is $1.38 \mathrm{~A}$, which corresponds to about $94 \%$ ionization of the injected neutral flux. The time averaged electron current entering the channel is therefore about 0.6 A. This value is obviously dependent on the choice of anomalous transport parameters. The calculated thrust and thrust efficiency are, respectively, $30.2 \mathrm{mN}$ and $46 \%$. Here and in the rest of this paper, the thruster efficiency is defined as $\eta=\frac{1}{2} \frac{T^{2}}{\dot{m}_{a} P_{T}}$, where $T$ is the thrust and $P_{T}=\left(V_{D C} I_{T}+P_{R F}\right)$ the total electric power absorbed in the thruster including the DC discharge power and the RF power (we do not include here the power dissipated in the cathode).

The spatial distribution of the electric potential for a DC voltage, $\mathrm{V}_{\mathrm{DC}}=250 \mathrm{~V}$, and no $\mathrm{RF}$ power in the ionization stage, $\mathrm{P}_{\mathrm{RF}}=0$ (operation in a single stage regime), is shown in Fig. 10. The potential drop is distributed in the magnetic barrier, as expected in a single stage thruster.

Since the maximum radial magnetic field is located in the middle of the channel (Fig. 8), the potential drop is also inside the channel. Because the shape of the equipotential contours follows more or less the magnetic lens defined by the magnetic barrier, this can lead to fast erosion of the channel walls. The channel length and profile will be modified in future prototypes to limit wall erosion.

The position of the anode can influence the plasma properties and thruster performance. The electric potential of Fig. 10 has been obtained with an anode ring located $1 \mathrm{~cm}$ from 


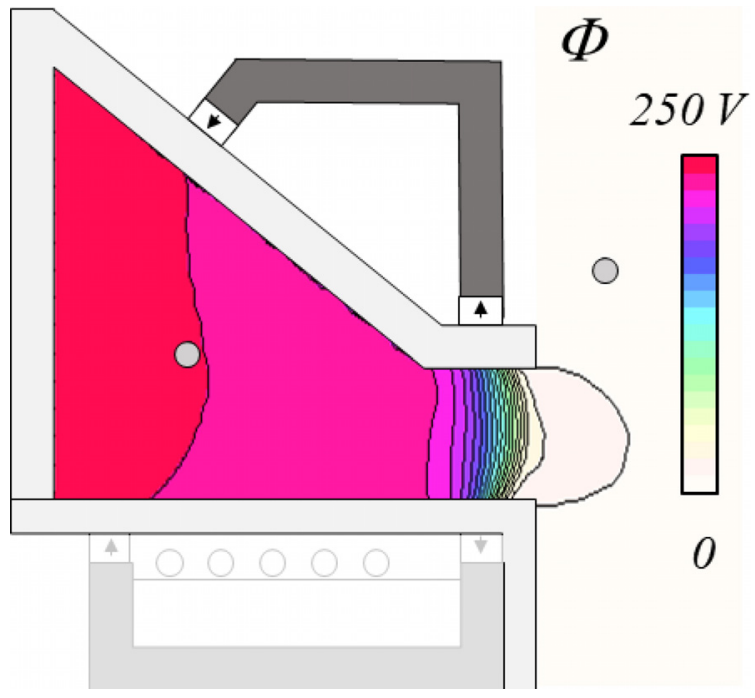

FIG. 10. Space distribution of the electric potential for $\mathrm{V}_{\mathrm{DC}}=250 \mathrm{~V}$, $\mathrm{P}_{\mathrm{RF}}=0$, and for a xenon mass flow rate of $2 \mathrm{mg} / \mathrm{s}$. The anode ring is located $1 \mathrm{~cm}$ from the back plate of the ionization stage.

the back plate of the ionization chamber (see Fig. 8). If the anode is moved closer to the back plate, e.g., at $3 \mathrm{~mm}$ instead of $1 \mathrm{~cm}$, the potential profile in the ionization chamber is significantly modified and is no longer flat. The potential is lower in the right part of the ionization chamber and increases between plasma and anode (see Fig. 11). This is because in that case, the anode is located in the large magnetic field region of the back cusp (Fig. 8). Since the electron conductivity is low in this region, the axial electric field must increase to draw the electron current to the anode (this region acts as a second magnetic barrier). If the plasma density in the ionization chamber is increased by turning on the $\mathrm{RF}$ power $\left(\mathrm{P}_{\mathrm{RF}}=100 \mathrm{~W}\right.$ in the example of Fig. 11), the electron conductivity increases in this region and the potential drop in the anode region disappears. Note that when operating in a double-stage regime, the anode must be close to the back plate to ensure efficient trapping of the electrons heated by the RF source.

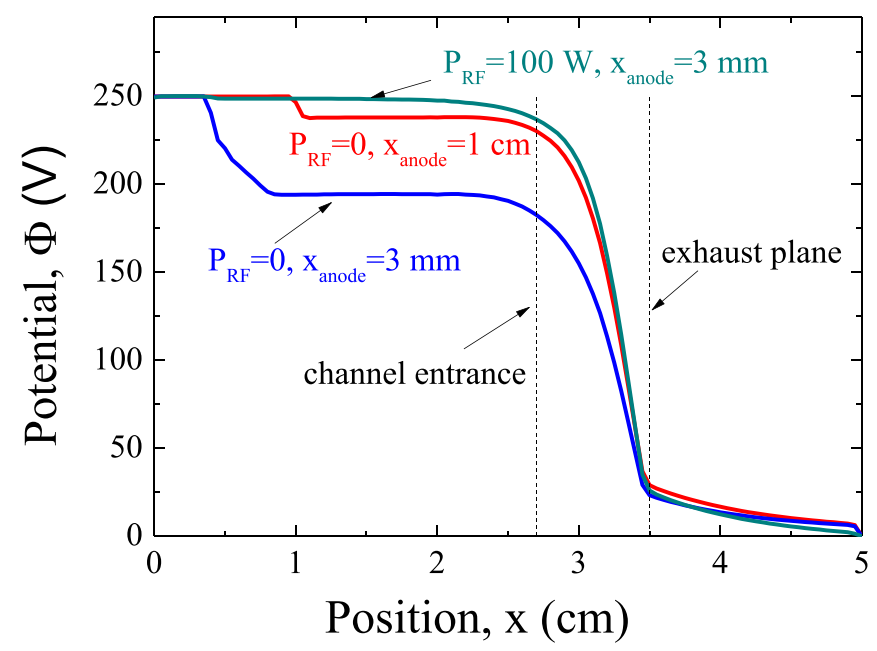

FIG. 11. Axial profiles of the electric potential along a line passing in the middle of the channel, for $\mathrm{V}_{\mathrm{DC}}=250 \mathrm{~V}$ and for 3 conditions: no RF power and anode at $1 \mathrm{~cm}$ from the back plate (as in Fig. 10), no RF power and anode at $3 \mathrm{~mm}$ from the backplate, and $\mathrm{P}_{\mathrm{RF}}=100 \mathrm{~W}$ and anode at $3 \mathrm{~mm}$.
Figure 12 shows the spatial distribution of the plasma density, atom density, electron mean energy, and ionization rate in the ionization chamber and thruster channel in the conditions of Fig. 10. The maximum plasma density, on the order of $2 \times 10^{18} \mathrm{~m}^{-3}$, is consistent with what is expected in a single stage Hall thruster and is located at the entrance of the channel. The plasma density presents a relative minimum between the thruster channel and the ionization chamber, and a second maximum in the chamber, at about $1.5 \times 10^{18} \mathrm{~m}^{-3}$. This is due to the confinement by the cusped magnetic field (the plasma density in the ionization chamber is maximum around the zero magnetic field point).

The maximum atom density close to the injection plane is on the order of $10^{20} \mathrm{~m}^{-3}$. As said above and seen on the atom density plot, a large part of the neutral flux is ionized in the channel. The maximum electron mean energy is about $50 \mathrm{eV}$ and is located, as expected in a single stage thruster, on the upstream side of the magnetic barrier. The electron mean energy decreases to less than $5 \mathrm{eV}$ in the ionization chamber. The ionization rate is maximum at the entrance of the thruster channel, a few mm upstream of the maximum radial magnetic field in the acceleration stage. The plasma properties and performance of the thruster in a single stage mode of operation, as predicted by the model, are rather satisfactory and seem good enough for this experimental thruster (no attempt has been made to optimize performance or lifetime). Of course, the presented results are dependent on the values of the adjustable transport parameters and these first results must be validated by comparisons with experiments.

\section{Double stage operation}

In this section, we illustrate the double stage regime of operation under conditions where the applied DC voltage is

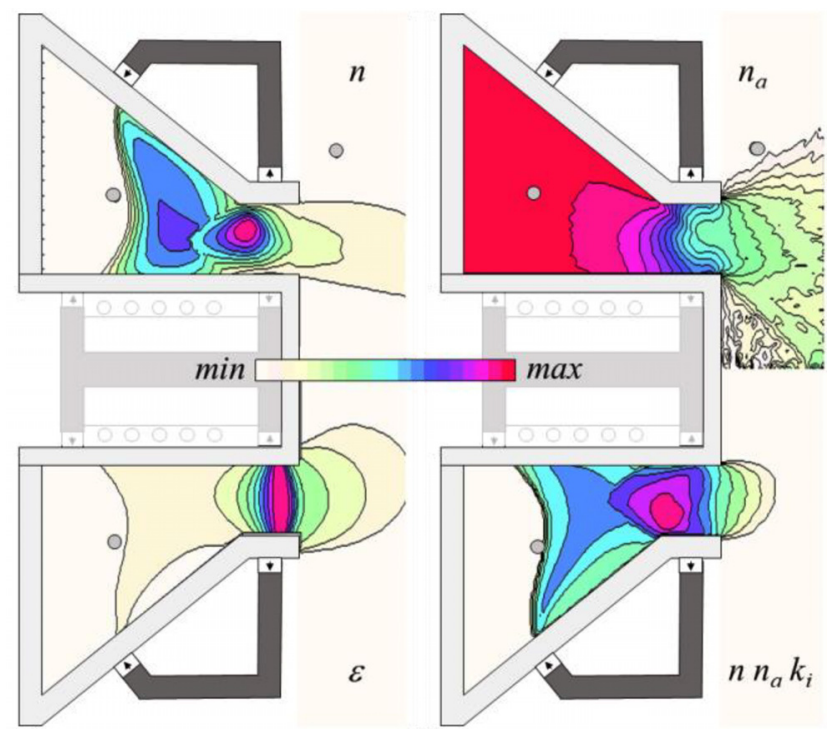

FIG. 12. Space distributions of the plasma density, $n$, atom density, $n_{a}$, electron mean energy, $\varepsilon$, and ionization rate, $S=n n_{a} k_{i}$ in the conditions of Fig. $10\left(\mathrm{~V}_{\mathrm{DC}}=250 \mathrm{~V}, \mathrm{P}_{\mathrm{RF}}=0,2 \mathrm{mg} / \mathrm{s}\right.$ xenon mass flow rate $)$. The anode is located $1 \mathrm{~cm}$ from the back plate. The plasma density $n\left(\left[0,2 \times 10^{18} \mathrm{~m}^{-3}\right]\right.$ and mean electron energy $\varepsilon([0,50 \mathrm{eV}])$ are plotted in the linear scale while the atom density $n_{a}$ (maximum $10^{20} \mathrm{~m}^{-3}$ ) and ionization rate $S=n n_{a} k_{i}$ (maximum $2.5 \times 10^{24} \mathrm{~m}^{3} \mathrm{~s}^{-1}$ ) are plotted on a 3 decades log scale. 
relatively low, $\mathrm{V}_{\mathrm{DC}}=100 \mathrm{~V}$ and for an $\mathrm{RF}$ power $\mathrm{P}_{\mathrm{RF}}$ $=200 \mathrm{~W}$, and a xenon mass flow rate of $2 \mathrm{mg} / \mathrm{s}$. The anode is located $3 \mathrm{~mm}$ from the back plate.

In these conditions, the calculated total current is about $1.5 \mathrm{~A}, 83 \%$ of the neutral flux is ionized, i.e., the extracted ion current is $\mathrm{I}_{\mathrm{i}}=0.83 \times 1.47=1.22 \mathrm{~A}$ and the electron current entering the channel is $0.25 \mathrm{~A}$. The total power is about $350 \mathrm{~W}$ ( $200 \mathrm{~W}$ from the RF coupling in the ionization stage, and $150 \mathrm{~W}$ due to the applied DC voltage). The calculated thrust is $18 \mathrm{mN}$ and the efficiency is $23 \%$.

The space distribution of electric potential is shown in Fig. 13. The whole potential drop takes place in the magnetic barrier of the acceleration stage. There is no potential drop on the anode side in spite of the anode location in the large magnetic field region close to the back plate $(3 \mathrm{~mm})$ of the ionization chamber. This is due to the large ionization and plasma density in the chamber due to the absorbed RF power, resulting in a large electron conductivity in the anode region.

Spatial distributions of the plasma density, atom density, mean electron energy, and ionization rate are displayed in Fig. 14. The plasma density is maximum (on the order of $6 \times 10^{18} \mathrm{~m}^{-3}$ ) next to the outer wall of the ionization chamber and decreases toward the chamber axis and channel entrance.

The calculated total ion losses to the walls and anode in the conditions of Fig. 14 correspond to a current of $2.7 \mathrm{~A}$, to be compared with the extracted ion current of about $1.2 \mathrm{~A}$. The wall losses are therefore not negligible and optimization of the cusp magnetic field is needed to reduce the losses.

There is a large depletion of the neutral density due to ionization in a relatively large volume of the ionization chamber upstream of the channel entrance. A large part of the ionization is performed in the ionization stage by electrons heated by the RF inductive source.

The ionization rate $S$ in the first stage is proportional to the neutral density and is maximum on the edge of the neutral depletion. This explains the maximum plasma density

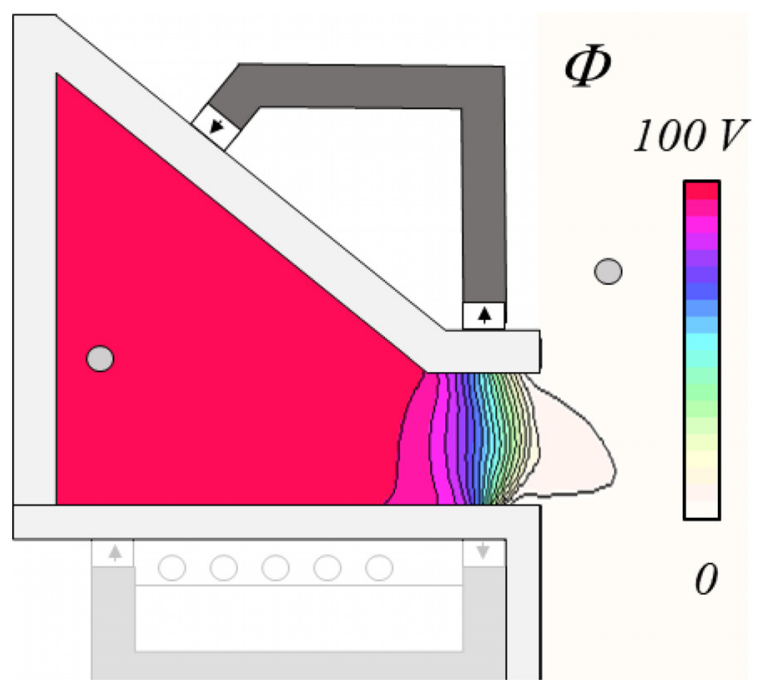

FIG. 13. Distribution of the electric potential in a double stage regime of operation: $\mathrm{V}_{\mathrm{DC}}=100 \mathrm{~V}, \mathrm{P}_{\mathrm{RF}}=200 \mathrm{~W}$, and a xenon mass flow rate of $2 \mathrm{mg}$ / $\mathrm{s}$. The anode is located $3 \mathrm{~mm}$ from the back plate.

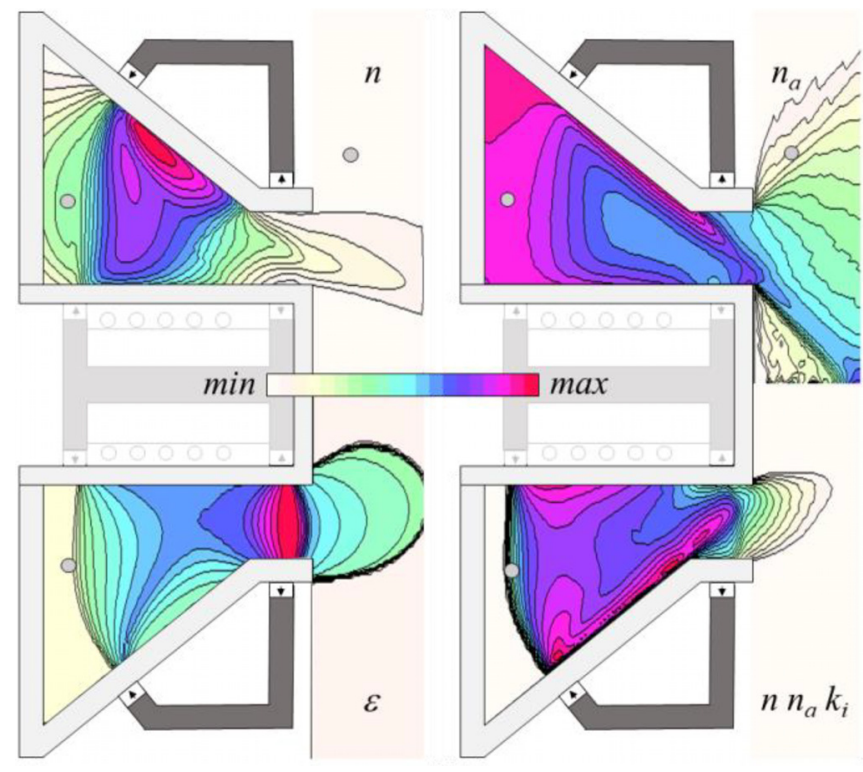

FIG. 14. Contour plots of the plasma density, $n$, atom density, $n_{a}$, electron mean energy, $\varepsilon$, and ionization rate, $S=n n_{a} k_{i}$ in the conditions of Fig. 13 $\left(\mathrm{V}_{\mathrm{DC}}=100 \mathrm{~V}, \mathrm{P}_{\mathrm{RF}}=200 \mathrm{~W}, 2 \mathrm{mg} / \mathrm{s}\right.$ xenon mass flow rate, the anode is located $3 \mathrm{~mm}$ from the back plate). The plasma density $n\left[0,6 \times 10^{18} \mathrm{~m}^{-3}\right]$ and mean electron energy $\varepsilon([0,13 \mathrm{eV}])$ are plotted in the linear scale while the atom density $n_{a}$ (maximum $10^{20} \mathrm{~m}^{-3}$ ) and ionization rate $S=n n_{a} k_{i}$ (maximum $2 \times 10^{24} \mathrm{~m}^{-3} \mathrm{~s}^{-1}$ ) are plotted on a 3 decades log scale.

along the outer wall of the ionization chamber. Note that the peak of neutral atom density along the outer wall of the ionization stage continues inside the channel, leading to a relative maximum of atom density along the channel axis.

The electron mean energy in the channel is, as expected, significantly lower than in the case $\mathrm{V}_{\mathrm{DC}}=250 \mathrm{~V}$ (maximum on the order of $13 \mathrm{eV}$ compared with $50 \mathrm{eV}$ for a DC voltage of $250 \mathrm{~V}$, see Fig. 12). For $\mathrm{V}_{\mathrm{DC}}=100 \mathrm{~V}$ and $\mathrm{P}_{\mathrm{RF}}=200 \mathrm{~W}$ (Fig. 14), the mean energy in the zero B-field region of the ionization chamber is on the order of $8 \mathrm{eV}$. The electron temperature in the ionization chamber adjusts so that charged particle losses to the walls (including the anode and channel) are balanced by ionization. The atom density in the chamber decreases with increasing absorbed power and the electron temperature must increase to maintain sufficient ionization to balance the losses. For $\mathrm{V}_{\mathrm{DC}}=100 \mathrm{~V}$, the mean energy around the zero B field in the chamber is, respectively, 6, 8, and $12 \mathrm{eV}$ for $\mathrm{P}_{\mathrm{RF}}$ equal to 100,200 , and $300 \mathrm{~W}$. The electron energy loss rates due to collisions with atoms $\left(n_{a} \kappa\right.$ term in the energy equation above) and due to electron-wall collisions ( $W$ term) are on the same order of magnitude in the conditions of Fig. 14: maximum value $1.4 \times 10^{7} \mathrm{eV} / \mathrm{s}$ in the channel, and a spatially averaged value of about $3 \times 10^{6} \mathrm{eV} /$ $\mathrm{s}$ in the chamber.

\section{Parametric study}

In this section, we analyze the global properties and performance of ID-HALL for different values of the DC voltage of the acceleration stage and RF power in the ionization stage, and for a fixed xenon mass flow rate of $2 \mathrm{mg} / \mathrm{s}$.

Figure 15 displays the calculated thrust as a function of total power $(\mathrm{DC}+\mathrm{RF})$ for three values of the $\mathrm{DC}$ voltage 


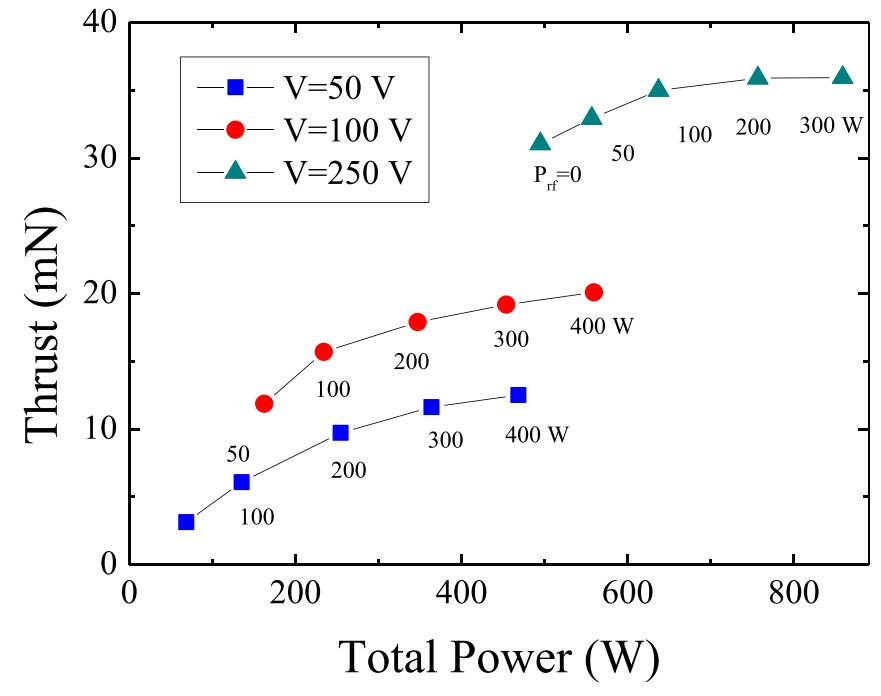

FIG. 15. Calculated thrust as a function of total power for three values of the applied DC voltage $(50,100$, and $250 \mathrm{~V})$ and a xenon mass flow rate of $2 \mathrm{mg} / \mathrm{s}$. Each point corresponds to a given value of the absorbed RF power.

(50, 100, and $250 \mathrm{~V})$, and Fig. 16 shows the thrust per unit power in $\mathrm{mN} / \mathrm{kW}$. The value of the RF power is indicated on each point of the curves. The thrust is defined as $T=\dot{m}_{i} v_{i}$, where $\dot{m}_{i}$ is the ion mass flow rate and $v_{i}$ is the axial mean ion velocity. If the neutral flux is fully ionized, the ion mass flow rate is equal to the gas mass flow rate $(2 \mathrm{mg} / \mathrm{s}$ in these simulations) and the thrust is simply proportional to the ion beam velocity, i.e., roughly proportional to the square root of the applied voltage. It is therefore not surprising that the thrust increases with the DC voltage in Fig. 15. Note that in the low DC voltage cases, $\mathrm{V}_{\mathrm{DC}}=50 \mathrm{~V}$, and $\mathrm{V}_{\mathrm{DC}}=100 \mathrm{~V}$, the thrust significantly increases with increasing RF power. This is because $\dot{m}_{i}$ increases, i.e., the fraction $I_{i} / I_{a}=\dot{m}_{i} / \dot{m}_{a}$ of the neutral flow ionized in the RF chamber increases with RF power $\left(I_{i}\right.$ is the extracted ion current and $I_{a}=e \dot{m}_{a} / m_{a}$, is the equivalent neutral atom current: $I_{a}=1.47 \mathrm{~A}$ for xenon with a mass flow rate $\dot{m}_{a}=2 \mathrm{mg} / \mathrm{s}$ ), as can be seen in Fig. 17. For a given DC voltage, the thrust reaches an asymptotic

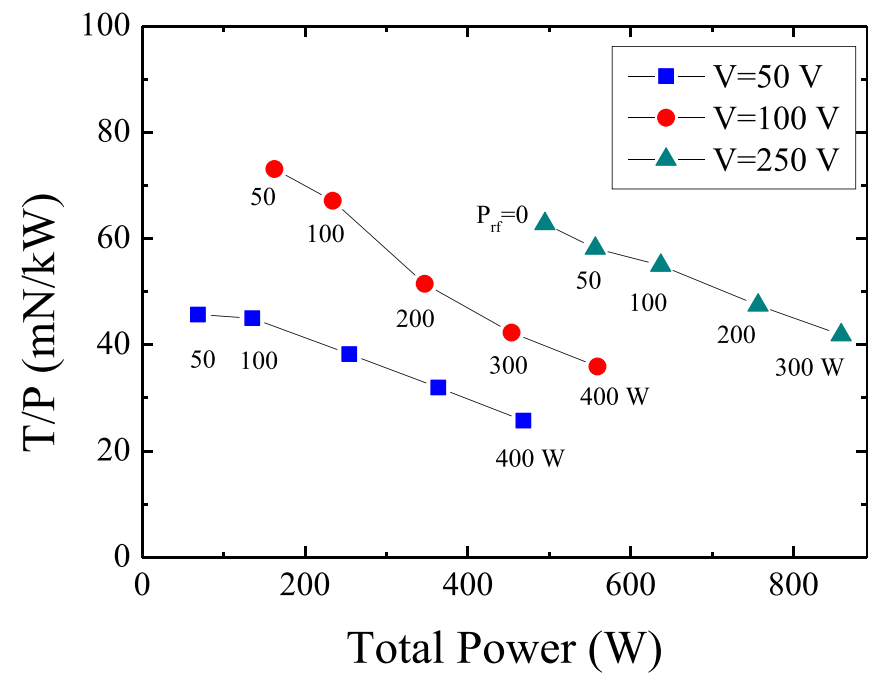

FIG. 16. Calculated thrust per unit power, in $\mathrm{mN} / \mathrm{kW}$ as a function of total power in the conditions of Fig. 15.

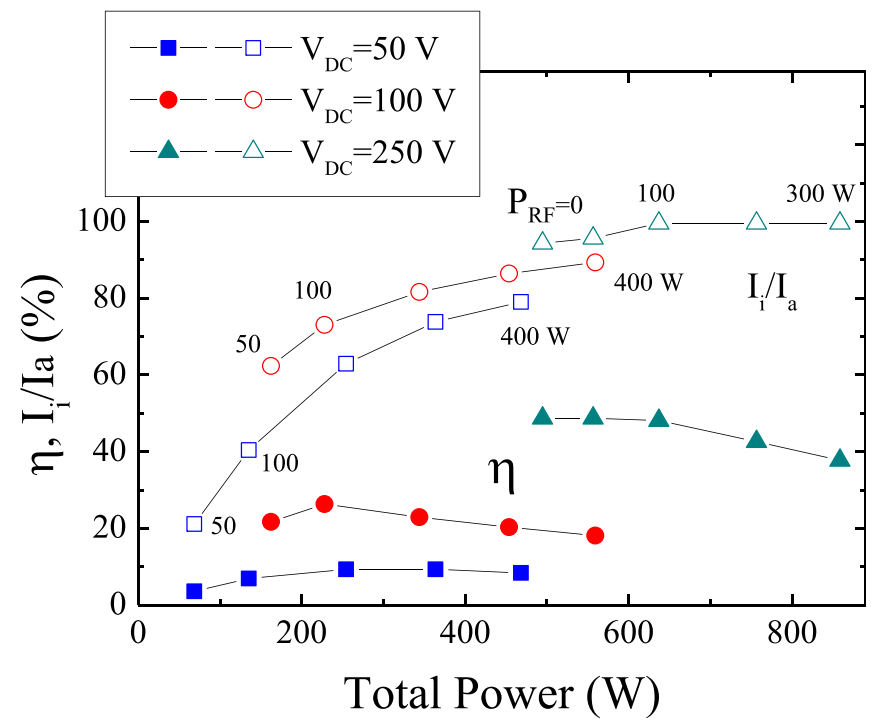

FIG. 17. Fraction $I_{i} / I_{a}$ of the neutral flow ionized in the thruster (open symbols), and thruster efficiency $\eta$ (full symbols) as a function of total power for three values of the DC voltage $(50,100$, and $250 \mathrm{~V})$ and a xenon mass flow rate of $2 \mathrm{mg} / \mathrm{s}$. Same conditions as Figs. 15 and 16 .

value at high $\mathrm{RF}$ powers because the maximum ion mean velocity is defined by the DC voltage (we neglect multiple charged ions) and the neutral flow becomes fully ionized $\left(\dot{m}_{i} \rightarrow \dot{m}_{a}\right)$.

Since the thrust saturates at high power for a given DC voltage, the thrust per unit power decreases as a function of total power as can be seen in Fig. 16.

The thruster efficiency $\eta$, defined above, is displayed in Fig. 17 as a function of total power for the three values of the DC voltage and different values of the RF power in the ionization chamber.

We first see that for DC voltages that are not sufficient to maintain a discharge (typically below $100 \mathrm{~V}$ in the simulations), the RF source in the ionization chamber allows discharge ignition and sustainment. In this voltage range and for sufficiently large RF powers, a large part of the neutral flow can be ionized in the ionization stage (more than $70 \%$ at $\mathrm{P}_{\mathrm{RF}}=300 \mathrm{~W}$ and $\mathrm{V}_{\mathrm{DC}}=50 \mathrm{~V}$, and more than $80 \%$ at $300 \mathrm{~W}$ and $100 \mathrm{~V}$ ). At $250 \mathrm{~V}$, the RF power is not really useful since a large part of the neutral flow is ionized due to the DC power.

Therefore, $T$ and $I_{i} / I_{a}$ do not significantly increase with increasing RF power at high voltages (for the chosen value of the xenon mass flow rate), and the efficiency $\eta$ decreases with increasing power.

At lower voltages, even though $I_{i} / I_{a}$ can be large at large RF powers, the thruster efficiency is smaller because the thrust is smaller (Fig. 15) due to the smaller ion beam velocity.

\section{SENSITIVITY OF THE RESULTS TO THE ANOMALOUS TRANSPORT COEFFICIENTS}

As discussed in the model description of Sec. V, electron transport across the magnetic field is artificially increased by introducing anomalous transport coefficients based on effective collision frequencies, $\nu_{w}$ for electron-wall 
interaction, and $\nu_{B}$ for turbulence. In the results of Sec. V, these effective collision frequencies were supposed to be due to wall collisions inside the thruster (channel and ionization stage), of the form $\nu_{w}=\alpha \times 10^{7} \mathrm{~s}^{-1}$, with $\alpha=1$, and to turbulence outside the thruster, $\nu_{B}=\beta / 16 \times e B / m_{e}$, with $\beta$ $=1\left(\nu_{B}=0\right.$ inside the thruster and $\nu_{w}=0$ outside $)$. The energy loss frequency due to electron-wall interaction was supposed to be of the form $\nu_{\varepsilon}=\delta \times 10^{7} \exp \left(-U / \varepsilon_{e}\right) \mathrm{s}^{-1}$ with $\delta=0.5$ inside the channel and ionization chamber and $\delta=0$ outside the thruster, and $U=20 \mathrm{eV}$.

In a single stage Hall thruster, Bareilles et al. ${ }^{30}$ noted that although the regime of current oscillations and some of the plasma properties may be strongly dependent on the anomalous transport coefficients, the overall performance are less sensitive provided that these coefficients are within a "reasonable" range (for example, anomalous transport must be much larger outside the exhaust plane than in the channel). In a DSHT, there are other uncertainties related to possible instabilities or turbulence and electron energy losses due to wall interaction in the ionization chamber. In the results of Sec. V, the anomalous momentum and energy collision frequencies in the channel were supposed to be the same as in the channel. This certainly overestimates the energy loss frequency in the chamber since the presence of the cusps and the lower electron temperature should lead to smaller energy losses. In order to analyze the sensitivity of the results to the choice of the effective momentum and energy losses, we considered the opposite case of no momentum and energy loss in the chamber.

Figure 18 shows a comparison between the thrust efficiency $\eta$ and the ionization efficiency $I_{i} / I_{a}$ as a function of total power for two values of the $\alpha$ coefficient in the channel ( $\alpha=1$, as above, and $\alpha=0.5$ ) and for two assumptions on the electron momentum and energy losses in the chamber: (1) the same as in the channel (i.e., $\alpha=1$ or $\alpha=0.5$ ) for the momentum losses and $\delta=0.5$, as above, for the energy losses and (2) no momentum and energy losses in the chamber, $\alpha=\delta=0$. The $\beta$ coefficient outside the thruster is equal to 1 in all cases. The limit between the channel and the ionization chamber in this model is defined by the magnetic field lines going from the walls next to the channel entrance to the point of zero magnetic field in the chamber.

We see in Fig. 18 that the differences between the $\alpha=1$ and $\alpha=0.5$ cases are relatively small. The differences between losses or no losses in the ionization chamber are more important. When momentum and energy losses in the chamber are zero, the thrust efficiency and ionization efficiency exhibit some maxima for $P_{R F}$ on the order of $100 \mathrm{~W}$. In the cases of no losses in the chamber, the maximum thrust efficiency and ionization efficiency are on the order of $30 \%$ and $90 \%$, respectively. For values of $P_{R F}$ larger than $100 \mathrm{~W}$, the thrust efficiency decreases because the extra power does not lead to more efficient ionization.

Above $P_{R F}=100 \mathrm{~W}$, ionization increases in the chamber and the atom density decreases but a larger number of ions recombine on the walls next to the channel entrance, which actually leads to a decrease in the overall ionization efficiency.
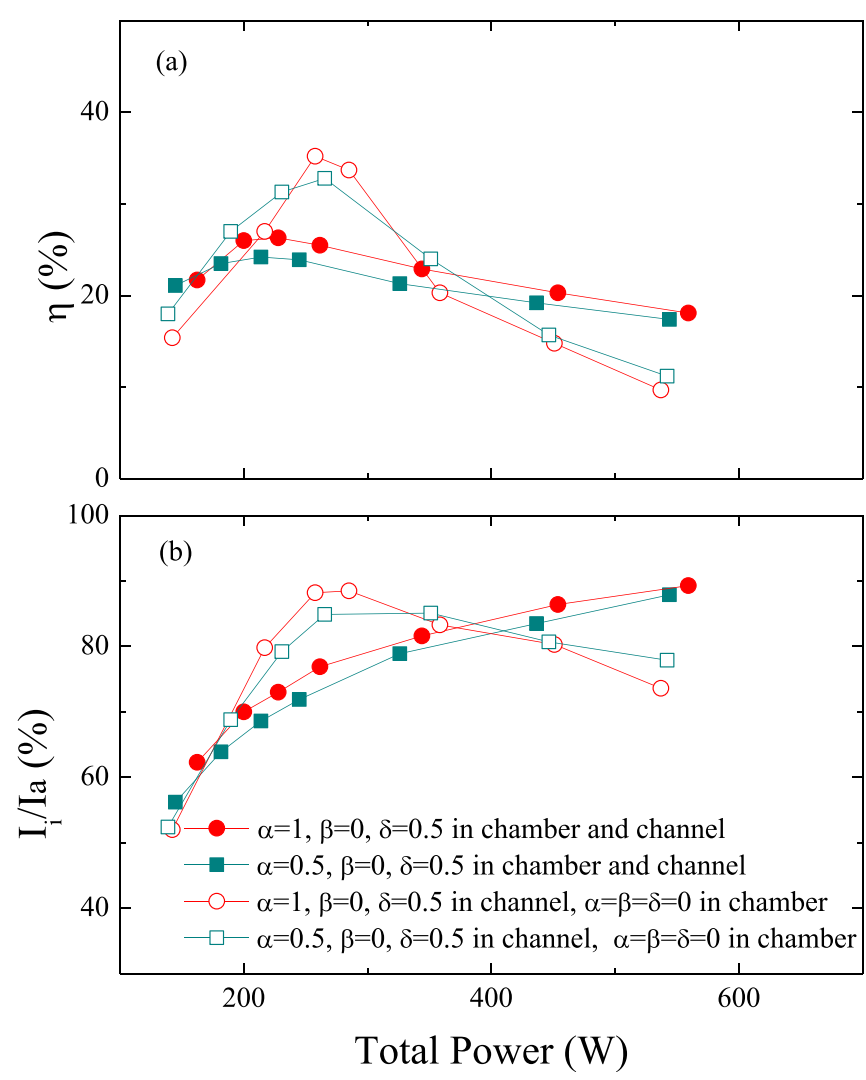

FIG. 18. (a) Thrust efficiency $\eta$ and (b) ionization efficiency (or mass utilization efficiency) $I_{i} / I_{a}$ as a function of total power for $V_{D C}=100 \mathrm{~V}$ (and different values of $P_{R F}$ ) and for different assumptions on anomalous transport. Full symbols: $\alpha=1$ (circles), and $\alpha=0.5$ (rectangles), with $\delta=0.5$, inside the channel and in the ionization chamber; hollow symbols: $\alpha=1$ (circles), and $\alpha=0.5$ (rectangles) inside the channel only and no anomalous transport in the ionization chamber. In all cases, the anomalous coefficients outside the thruster are the same: $\alpha=0, \beta=1$, and $\delta=0$. The different points (from left to right) on each curve correspond to $P_{R F}=50,75,100$, $125,200,300$, and $400 \mathrm{~W}$.

Since the atom density decreases with increasing $P_{R F}$ in the chamber, the maximum electron mean energy in the chamber increases with $P_{R F}$ and reaches $12 \mathrm{eV}$ at $P_{R F}=100 \mathrm{~W}$, $32 \mathrm{eV}$ at $125 \mathrm{~W}$, and $45 \mathrm{eV}$ at $200 \mathrm{~W}$ (when losses are included the chamber, with $\alpha=1$ and $\delta=0.5$, the maximum electron mean energy in the chamber at $200 \mathrm{~W}$ was $8 \mathrm{eV}$ ).

The results above show that (1) the assumptions of the electron momentum and energy losses in the chamber strongly affect the plasma properties, (2) the maximum thrust efficiency is in the range [20\%-30\%] for the two extreme assumptions of losses and no-losses in the chamber (with an ionization efficiency in the range $70 \%-90 \%$ ), and (3) there is an optimum RF power for a given mass flow rate, which is on the order of $100 \mathrm{~W}$ for our conditions of $2 \mathrm{mg} / \mathrm{s}$ xenon flow rate and $100 \mathrm{~V}$ DC voltage.

Figure 19 compares the axial profiles of the "real" and "anomalous" electron collision frequencies at the steady state at $\mathrm{VDC}=100 \mathrm{~V}$, for $\mathrm{P}_{\mathrm{RF}}=200 \mathrm{~W}$ assuming momentum and energy losses in the ionization chamber [Fig. 19(a)], and for $\mathrm{P}_{\mathrm{RF}}=100 \mathrm{~W}$ with no momentum and energy losses in the ionization chamber [Fig. 19(b)]. Figure 19(a) corresponds to the case of Fig. 14 where the anomalous electron momentum and energy collision frequencies are supposed to 


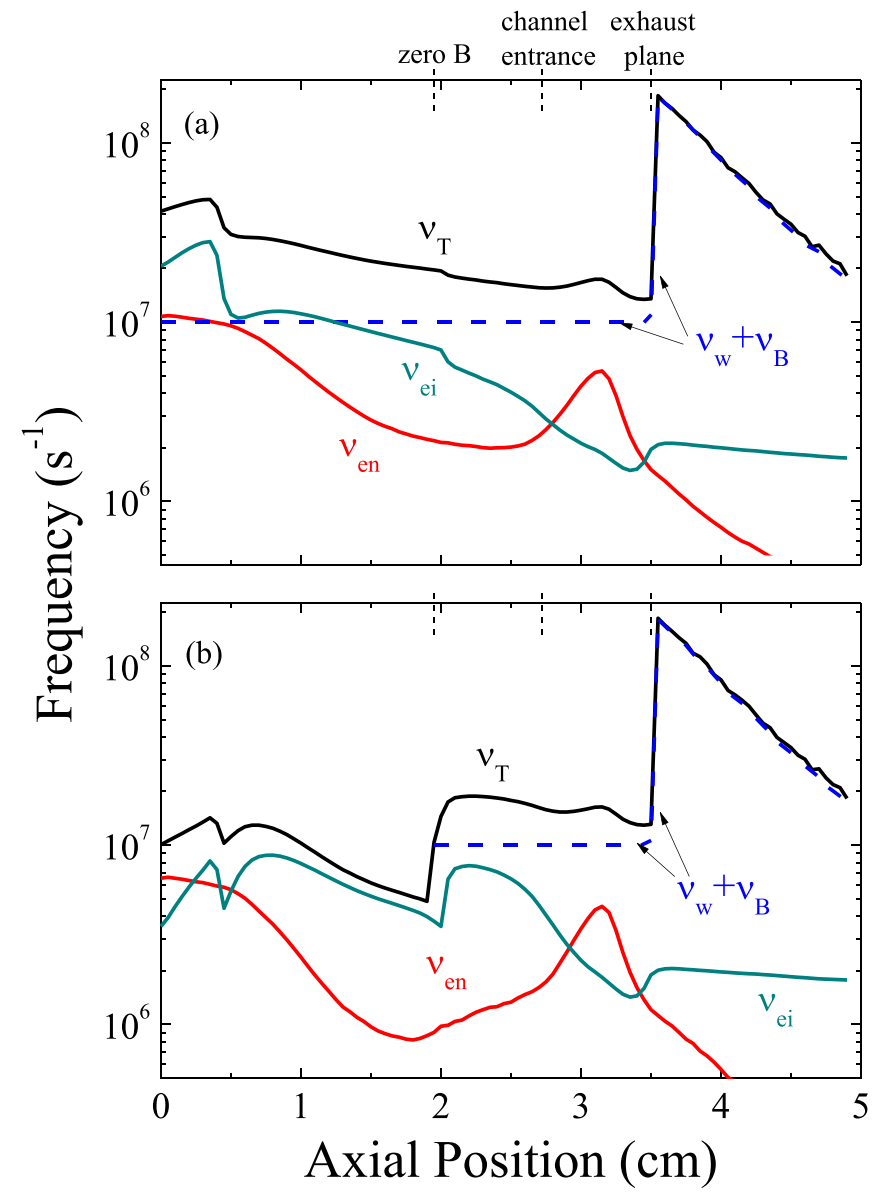

FIG. 19. Axial profiles (along the mid-channel line) of the electron-neutral $\left(\nu_{\mathrm{en}}\right)$, electron-ion $\left(\nu_{\mathrm{ei}}\right)$, anomalous $\left(\nu_{\mathrm{w}}+\nu_{\mathrm{B}}\right)$, and total $\left(\nu_{\mathrm{T}}\right)$, collision frequencies, for a xenon mass flow rate of $2 \mathrm{mg} / \mathrm{s}$ and $\mathrm{V}_{\mathrm{DC}}=100 \mathrm{~V}$, (a) for a $\mathrm{RF}$ power of $200 \mathrm{~W}$ and anomalous transport defined by $\alpha=1, \beta=0$, and $\delta=0.5$, inside the channel and in the ionization chamber, and $\alpha=0, \beta=1$, and $\delta=0$ outside the thruster, (b) for a RF power of $100 \mathrm{~W}$ and $\alpha=1$, $\beta=0$, and $\delta=0.5$, inside the channel, $\alpha=0, \beta=0$, and $\delta=0$ in the ionization chamber, and $\alpha=0, \beta=1$ and $\delta=0$ outside the thruster. The axial positions of the zero magnetic field point, channel entrance, and exhaust plane are indicated by the dashed lines.

be constant inside the channel and in the chamber $(\alpha=1, \beta$ $=0$, and $\delta=0.5$ ), while the anomalous collision frequency outside the thruster is of the Bohm type $(\alpha=0, \beta=1$, and $\delta=0)$. This choice of anomalous transport parameters leads to a sharp increase in the effective collision frequency and electron mobility around the channel exhaust, which generally leads to a better fit of experimental results (see, e.g., Ref. 33). We see in both cases a large depletion of the neutral density due to ionization in the chamber. The depletion is even larger in the case of Fig. 19(b) where the absorbed RF power is twice lower than in the case of Fig. 19(a). This is because some energy losses due to electron-wall interactions are taken into account (and probably overestimated) in Fig. 19(a). The electron-neutral momentum transfer frequency increases around the channel entrance due to the increase in the atom density associated with ion recombination at the walls. Finally note that the electron-ion Coulomb collision frequency in the ionization chamber is much larger than the electron-neutral collision frequency and can contribute to cross-field transport as much as the anomalous collision frequency of $10^{7} \mathrm{~s}^{-1}(\alpha=1)$. The differences between the results displayed in Figs. 19(a) and 19(b) (and between the full symbols and open symbols curves of Fig. 18) are due to the difference on the energy losses assumption in the chamber more than to the difference in momentum losses.

\section{CONCLUSION}

The aim of this paper was to study the relevance of the Double Stage Hall Thruster concept and to propose an optimized design.

The proposed design, ID-HALL (patent pending), combines an inductively coupled ionization stage with a coil inside the inner cylinder of the thruster, which can be easily and efficiently connected to the magnetic barrier of the acceleration stage. ID-HALL is designed to allow an efficient ionization in the plasma source, to minimize losses to the walls, and to generate ions as close as possible to the accelerating field. These last two criteria were not met in some previous designs described in the literature.

The results of the simulations of ID-HALL operations using a 2D hybrid model are summarized below. Although the model results rely on the assumptions on the electron anomalous transport coefficients (a non-exhaustive sensitivity analysis is provided), we believe that the model can provide useful insight on the physics of the thruster, on the trends that can be expected when parameters such as DC voltage ore RF power are varied, and on the ability of the thruster to operate as a DSHT. According to the model predictions, the thruster seems to operate satisfactorily in a single stage mode, with performance roughly comparable to Hall thrusters of the same size (this first experimental prototype has however not been optimized for important properties such as lifetime and beam divergence).

In double stage operation, the simulations indicate that the neutral flow can be efficiently ionized by the inductive plasma source for DC voltages close to or lower than the threshold voltage for DC operation. For a xenon mass flow rate of $2 \mathrm{mg} / \mathrm{s}$, more than $70 \%$ of the neutral flow is ionized at $50 \mathrm{~V} \mathrm{DC}$, with an RF power of $300 \mathrm{~W}$. This possibility of efficient ionization at low voltage and reasonable RF power is promising for applications to operation with gas lighter than xenon, such as krypton or argon (the mass of argon is almost 4 times smaller than that of xenon so that operation at the same specific impulse-or ion velocity must be performed at a DC voltage twice lower, i.e., generally too small for single stage regimes). ID-HALL operation will be tested in argon in future experiments and simulations.

Although the ionization region of the first stage is close to the acceleration region in the ID-HALL design, ion losses to the walls in the ionization chamber and in the transition region between the ionization stage and acceleration stage are still non-negligible. The magnetic field parallel to the walls and in the cusp regions of the ionization chamber should be optimized to reduce the losses.

At higher DC voltages, operation in a double stage mode is not useful if the neutral flow can be fully ionized by the electrons emitted by the external cathode and gaining energy in the acceleration region. This was clearly illustrated 
in the simulations by the example at $\mathrm{VDC}=250 \mathrm{~V}$ for a $2 \mathrm{mg} / \mathrm{s}$ xenon mass flow rate. Double stage operation for voltages larger than $200 \mathrm{~V}$ in xenon may be useful for larger mass flow rates if the neutral flow cannot be fully ionized in the acceleration stage. These situations will be explored in future simulations and experiments.

\section{ACKNOWLEDGMENTS}

This work was partially supported by the French Space Agency CNES and by the RTRA STAE foundation. L.D. benefits from a Ph.D. fellowship of the University of Toulouse III.

${ }^{1}$ D. M. Goebel and I. Katz, Fundamentals of Electric Propulsion: Ion and Hall Thrusters (Wiley, 2008).

${ }^{2}$ J.-P. Boeuf, J. Appl. Phys. 121, 011101 (2017).

${ }^{3}$ L. Dubois, F. Gaboriau, L. Liard, C. Boniface, and J. P. Boeuf, Phys. Plasmas 25, 093504 (2018).

${ }^{4}$ Y. Peterson, Ph.D. thesis, Michigan State University, 2004.

${ }^{5}$ A. I. Morozov, A. I. Bugrova, A. V. Desyatskov, V. K. Kharchenikov, M. Prioul, and L. Jolivet, in 28th International Electric Propulsion Conference, IEPC-2003-290, Toulouse, France (2003).

${ }^{6}$ A. I. Bugrova, A. V. Desiatskov, V. K. Kharchenikov, A. I. Morozov, and M. Prioul, in 29th International Electric Propulsion Conference, IEPC 2005-146, Princeton, NJ (2005).

${ }^{7}$ A. I. Morozov and V. V. Savelyev, Phys. Usp. 41, 1049 (1998).

${ }^{8}$ C. Boniface, G. Hagelaar, L. Garrigues, J. P. Boeuf, and M. Prioul, IEEE Trans. Plasma Sci. 33, 522 (2005).

${ }^{9}$ E. Chesta, D. Estublier, B. Fallis, E. Gengembre, J. Gonzalez del Amo, N. Kutufa, D. Nicolini, G. Saccoccia, L. Casalino, P. Dumazert, M. Prioul, J. P. Boeuf, A. Bouchoule, N. Wallace, G. Noci, M. Berti, M. Saverdi, L. Biagioni, S. Marcuccio, A. Cadiou, F. Darnon, and L. Jolivet, Acta Astronaut. 59, 931 (2006).

${ }^{10}$ L. Garrigues, C. Boniface, G. J. M. Hagelaar, and J. P. Boeuf, Phys. Plasmas 15, 113502 (2008).

${ }^{11}$ A. I. Bugrova, A. V. Desyatskov, V. K. Kharchenikov, and A. S. Lipatov, in 30th International Propulsion Conference, IEPC-2007-221, Florence, Italy (2007).

${ }^{12}$ D. Yu, M. Song, H. Li, H. Liu, and K. Han, Phys. Plasmas 19, 113505 (2012).

${ }^{13}$ D. Yu, M. Song, H. Liu, X. Zhang, and H. Li, Phys. Plasmas 19, 073511 (2012).
${ }^{14} \mathrm{P}$. Molina-Morales, H. Kuninaka, K. Toki, and Y. Arakawa, in 27th International Electric Propulsion Conference, IEPC-01-069, Pasadena, California, USA (2001).

${ }^{15}$ R. A. Martinez, W. A. Hoskins, P. Y. Peterson, and D. R. Massey, in 31st International Electric Propulsion Conference (IEPC), Ann Harbor, Mi (2009).

${ }^{16}$ Y. Peterson, D. R. Massey, A. Shabshelowitts, R. Shastry, and R. Liang, in 32nd International Electric Propulsion Conference, IEPC-2011-269, Kurhaus, Wiesbaden, Germany (2011).

${ }^{17}$ A. Shabshelowits and A. D. Gallimore, AIAA Paper 2012-4336, 2012.

${ }^{18}$ S. Harada, T. Baba, A. Uchigashima, S. Yokota, A. Iwakawa, A. Sasoh, T. Yamazaki, and H. Shimizu, Appl. Phys. Lett. 105, 194101 (2014).

${ }^{19}$ H. Kuwano, A. Ohno, H. Kuninaka, and H. Nakashima, in 30th International Electric Propulsion Conference, IEPC-2007-085, Florence, Italy (2007).

${ }^{20}$ A. I. Bugrova, G. E. Bugrov, V. K. Kharchenikov, M. I. Shaposhnikov, and S. Mazouffre, Tech. Phys. Lett. 38, 344 (2012).

${ }^{21}$ M. Cappaci, G. Matticari, G. E. Noci, P. Siciliano, M. Berti, L. Biagoni, U. Cesari, E. Gengembre, and E. Chesta, AIAA Paper 20043771, 2004.

${ }^{22}$ J. Perez-Luna, G. J. M. Hagelaar, L. Garrigues, and J. P. Boeuf, Phys. Plasmas 14, 113502 (2007).

${ }^{23}$ H. Kuwano, H. Nakashima, and H. Kuninaka, in Proceedings of the 24th International Symposium on Space Technology and Science, ISTS 2004, Miyazaki, Japan (2004)

${ }^{24}$ V. Godyak, J. Phys. D: Appl. Phys. 46, 283001 (2013).

${ }^{25}$ J. Arancibia Monreal, P. Chabert, and V. Godyak, Phys. Plasmas 20 , 103504 (2013).

${ }^{26}$ I. G. Mikellides, I. Katz, R. R. Hofer, and D. M. Goebel, J. Appl. Phys. 115, 043303 (2014).

${ }^{27}$ R. R. Hofer, D. M. Goebel, I. G. Mikellides, and I. Katz, J. Appl. Phys. 115, 043304 (2014).

${ }^{28} \mathrm{See}$ https://www.hallis-model.com for HALLIS software

${ }^{29}$ G. J. M. Hagelaar, J. Bareilles, L. Garrigues, and J. P. Boeuf, J. Appl. Phys. 91, 5592 (2002).

${ }^{30}$ J. Bareilles, G. J. M. Hagelaar, L. Garrigues, C. Boniface, J. P. Boeuf, and N. Gascon, Phys. Plasmas 11, 3035 (2004).

${ }^{31}$ J. Boeuf and L. Garrigues, J. Appl. Phys. 84, 3541 (1998).

${ }^{32}$ G. J. M. Hagelaar, J. Bareilles, L. Garrigues, and J. P. Boeuf, J. Appl. Phys. 93, 67 (2003).

${ }^{33}$ J. C. Adam, J. P. Boeuf, N. Dubuit, M. Dudeck, L. Garrigues, D. Gresillon, A. Heron, G. J. M. Hagelaar, V. Kulaev, N. Lemoine, S. Mazouffre, J. Perez Luna, V. Pisarev, and S. Tsikata, Plasma Phys. Controlled Fusion 50, 124041 (2008). 